\title{
A classification of spanning surfaces for alternating links
}

\author{
COLIN ADAMS \\ THOMAS KINDRED
}

\begin{abstract}
A classification of spanning surfaces for alternating links is provided up to genus, orientability, and a new invariant that we call aggregate slope. That is, given an alternating link, we determine all possible combinations of genus, orientability, and aggregate slope that a surface spanning that link can have. To this end, we describe a straightforward algorithm, much like Seifert's algorithm, through which to construct certain spanning surfaces called state surfaces, obtained by splitting each crossing one of the two ways, filling in the resulting circles with disks and connecting these disks with half twisted bands at the crossings. A particularly important subset of these will be what we call basic state surfaces. We can alter these surfaces by performing the entirely local operations of adding handles and/or crosscaps, each of which increases genus.
\end{abstract}

The main result then shows that if we are given an alternating projection $P(L)$ and a surface $S$ spanning $L$, we can construct a surface $T$ spanning $L$ with the same genus, orientability, and aggregate slope as $S$ that is a basic state surface with respect to $P$, except perhaps at a collection of added crosscaps and/or handles. Furthermore, $S$ must be connected if $L$ is nonsplittable.

This result has several useful corollaries. In particular, it allows for the determination of nonorientable genus for alternating links. It also can be used to show that mutancy of alternating links preserves nonorientable genus. And it allows one to prove that there are knots that have a pair of minimal nonorientable genus spanning surfaces, one boundary-incompressible and one boundary-compressible.

$57 \mathrm{M} 25$

\section{Introduction}

A Seifert surface for an oriented knot or link $L$ is a compact connected oriented surface in $S^{3}$ with oriented boundary equal to $L$. Seifert's algorithm, wherein one takes an oriented projection of the link, cuts each crossing open in the manner that preserves orientation, fills in each resulting circle with a disk, and connects the disks with a half-twisted band at each crossing, ensures that such a surface exists. The minimal 
genus of a Seifert surface is defined to be the genus of the knot or link. Given a random link, it is typically difficult to determine its genus. However for one category of link, we have a simple means for finding the genus.

Theorem 1.1 Given an oriented alternating link, Seifert's algorithm applied to a reduced alternating projection yields a minimal genus Seifert surface.

This theorem has been proven three times, first independently in 1958 by Kunio Murasugi [28; 29] and in 1959 by Richard Crowell [9] using the reduced Alexander Polynomial, and then in 1987 by David Gabai [14] using elementary geometric techniques. Our main theorem will include this theorem as a special case.

However, in this paper, we will also be concerned with nonorientable spanning surfaces. The crosscap number of a nonorientable surface $S$ of $n$ boundary components is defined to be $2-\chi(S)-n$. Note that this is the number of projective planes of which the corresponding closed surface is the connected sum. In Clark [8] (see also Murakami and Yasuhara [27]), the crosscap number of a link $L$ is defined to be the minimum crosscap number of any nonorientable surface spanning the link $L$.

For the sake of comparison between complexities of orientable and nonorientable surfaces, define the nonorientable genus of $L$ to be $\frac{1}{2}$ of the crosscap number of $L$. Note that this can be either an integer or a half integer, and does not coincide with traditional definitions. We will sometimes refer to the genus of a knot or link as the orientable genus if confusion may otherwise arise.

The crosscap number is known only for a relatively small collection of knots and links. In Teragaito [34], it was determined for torus knots (see also Mattman and Sizemore [20]). In Hirasawa and Teragaito [17], a simple method for its determination was provided for two-bridge knots and links. In Ichihara and Mizushima [19], it was determined for many pretzel knots. In a recent preprint (Burton and Ozlen [7]), the authors present an algorithm that utilizes normal surface theory and integer programming to determine the crosscap number for 27 new knots of 12 or fewer crossings and new upper bounds for many more.

In this paper, we present a classification of spanning surfaces for alternating links up to genus, orientability, and a new invariant that we call aggregate slope, and that for knots coincides with the slope. That is, given an alternating link, we determine all possible combinations of genus, orientability, and aggregate slope that a surface spanning that link can have. To this end, we describe a straightforward algorithm, much like Seifert's algorithm, through which to construct spanning surfaces. These surfaces are obtained by splitting the alternating knot projection at each crossing in one of the two ways. The 
resulting circles are then filled with disks, and the nested disks stacked in some order. Then the surface is constructed by attaching half-twisted bands across the crossings to obtain a surface spanning the knot. We call the surfaces generated by this algorithm state surfaces. A particularly important subset of these will be what we call basic state surfaces.

Once we have a state surface, we can also change the surface by performing the entirely local operations of adding handles and/or crosscaps, each of which increases genus.

Our main result is given here.

Theorem 5.4 Given an alternating projection $P(L)$ and a surface $S$ spanning $L$, there exists a surface $T$ spanning $L$ with the same genus, orientability, and aggregate slope as $S$ such that $T$ is a basic state surface with respect to $P$, except perhaps at a collection of added crosscaps and/or handles. When $S$ is orientable, $T$ can be chosen to be orientable with respect to the orientation that $L$ inherits from $S$.

This result has several useful corollaries that appear in Section 6, including the already mentioned classification of spanning surfaces for alternating links. Additionally, it allows us to easily find and construct a minimal nonorientable genus surface spanning a given alternating link. Note, for example in Zhang [37], that a substantial amount of work is necessary to show the crosscap number of the link $6_{3}^{2}$ is 3 . Theorem 5.4 and the algorithm given below make this an immediate conclusion. At the end of the paper, we include the nonorientable genus of all the alternating knots of nine or fewer crossings and alternating 2-component links of eight or fewer crossings. It would be straightforward to write a computer program to determine the nonorientable genus of all knots in the census of alternating knots through 22 crossings given in Hoste, Thistlethwaite and Weeks [18]; Rankin, Flint and Schermann [32] and [33].

Hatcher and Thurston [15] proved that a 2-bridge knot cannot have two minimal nonorientable genus spanning surfaces, one boundary-incompressible and one boundarycompressible. They then asked whether or not this is true in general. We utilize the results of this paper to answer that question in the negative with a specific example.

Recently, Curtis and Taylor [10] gave a further corollary of Theorem 5.4, showing that the two checkerboard surfaces of a reduced alternating projection of an alternating knot must yield the maximum and minimum integral slopes for all essential boundary surfaces of the knot. This implies that these maximum and minimum integral slopes must be twice the number of positive crossings and the negative of twice the number of negative crossings in the projection respectively. Since all slopes are integral for Montesinos knots, this yields the maximum and minimum slopes for all surfaces in that case. 
The state surfaces that appear here have independently been considered previously in Pabiniak, Przytycki and Sazdanović [31] (see footnote on page 10 of the ArXiv version) and in Ozawa [30], where a criterion is provided that proves certain such surfaces are essential. In more recent work, Futer [11] and Futer, Kalfagianni and Purcell [12; 13] determined the geometric type of state surfaces in hyperbolic knot exteriors. State surfaces continue to grow in importance.

\section{Background}

Standard definitions of reduced projections, flypes, positive and negative crossings, oriented links, incompressible and boundary-incompressible surfaces apply. A surface that is incompressible and boundary-incompressible is said to be essential.

If a projection $P$ contains $p$ positive crossings and $n$ negative crossings, then the writhe $w(P)$ is $p-n$. Writhe varies across projections of a given link and across orientations of a given projection, but is invariant across all reduced, alternating projections of an oriented link.

We define the aggregate linking number $\operatorname{lk}(L)$ to be the sum of the linking numbers of all pairs of link components. We define the aggregate linking number of a knot to be zero. Equivalently, the aggregate linking number can be calculated in the same way as writhe, but only counting crossings between distinct link components, and halving at the end. Linking number and aggregate linking number are invariant across all projections of a given oriented link, but may well vary across various orientations of a given link.

A projection divides the projection surface $S^{2}$ into regions, each of whose boundary consists alternately of strands and crossings from $L$. A region with $n$ crossings on its boundary will be called a projection $n-g o n$.

A spanning surface for a link $L$ is a surface $S \subset S^{3}$ with boundary equal to $L$ such that $S \cap \partial N(L)$ consists of one $(p, 1)$-curve on each component of $\partial N(L)$, where $p$ refers to the number of meridians. We will not distinguish between the spanning surface $S$ as a subset of $S^{3}$ and the spanning surface as a subset of $S^{3} \backslash N(L)$, calling both $S$. Two spanning surfaces for $L$ are considered equivalent if one is ambient isotopic to the other in $S^{3} \backslash N(L)$. We will assume that $S$ contains no closed components, so that every point in $S$ is connected to $L$ by a path in $S$.

We need a special form of boundary-incompressibility for spanning surfaces.

A spanning surface $S$ in $S^{3} \backslash N(L)$ is meridianally boundary-compressible if there exists a disk $D$ embedded in $S^{3} \backslash N(L)$ such that $\partial D=\alpha \cup \beta$, where $D \cap S=\beta$ and 
$D \cap \partial N(L)=\alpha$ are both arcs, $\beta$ does not cut a disk off $S, \partial D \cap \partial S$ cuts $\partial S$ into two $\operatorname{arcs} \phi_{1}$ and $\phi_{2}$, and $\alpha \cup \phi_{i}$ is a meridian of the link for at least one of $i=1,2$. A spanning surface is said to be meridianally boundary-incompressible if no such disk exists. The spanning surface is said to be meridianally essential if it is incompressible and meridianally boundary-incompressible.

The boundary slope or just slope $l(S, \hat{L})$ of a spanning surface $S$ with respect to a particular oriented component $\hat{L}$ of the link it spans is the linking number of $\hat{L}$ with $S \cap \partial N(\hat{L})$, its parallel curve on $S$ (where $S \cap \partial N(\hat{L})$ inherits the orientation of $\hat{L}$, as we will henceforth assume it to do). The aggregate slope $l(S, L)$ of a surface $S$ spanning oriented link $L$ is the sum of the link components' individual slopes. Note that slope and aggregate slope are independent of link orientation.

We define the twist of a spanning surface $S$ relative to projection $P$ to be $\tau(S, P)=$ $l(S, L)-(w(P)-2 \operatorname{lk}(L))$, where $l(S, L)$ is the aggregate slope, $w(P)$ is the writhe, and $\operatorname{lk}(L)$ is the aggregate linking number of $L$.

Proposition 2.1 The twist $\tau(S, P)$ can be calculated by taking the projection of $L \cup(S \cap \partial N(L))$ and considering only those crossings that occur between edges of the annuli in $S \cap N(L)$. If we have $p$ positive crossings of this type and $n$ negative crossings, then $\tau(S, P)=(p-n) / 2$.

Proof First, notice that $S \cap N(L)$ is a collection of annuli. Holding $L$ fixed, we can isotope each annulus in $S \cap N(L)$ so that in the projection of $L \cup(S \cap \partial N(L))$, any crossing between some component of $S \cap \partial N(L)$ and its respective boundary through an annulus in $S \cap N(L)$ does not occur in a neighborhood of a crossing of $L$ in the projection. The projection of $L \cup(S \cap \partial N(L))$ will then have the following seven types of crossings, where $L_{i}$ and $L_{j}$ denote any distinct pair of link components and $\hat{L}_{i}$ and $\hat{L}_{j}$ their respective components in $\hat{L}=S \cap \partial N(L)$ :

(I) $L_{i}$ with itself.

(II) $L_{i}$ with $L_{j}$.

(III) $L_{i}$ with $\hat{L}_{i}$, such that the preimage of the crossing is an arc connecting the two boundary components of the annulus $S \cap N\left(L_{i}\right)$.

(IV) $L_{i}$ with $\hat{L}_{i}$ in a neighborhood of a crossing of $L$, rather than through $S \cap N\left(L_{i}\right)$.

(V) $\hat{L}_{i}$ with itself, necessarily in the neighborhood of a crossing of $L$.

(VI) $L_{i}$ with $\hat{L}_{j}$, necessarily in the neighborhood of a crossing of $L$.

(VII) $\hat{L}_{i}$ with $\hat{L}_{j}$, necessarily in the neighborhood of a crossing of $L$. 


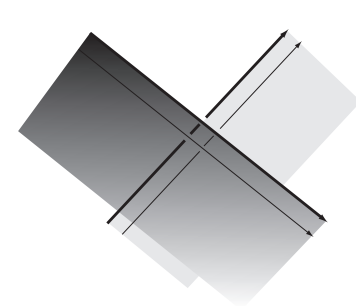

Figure 1: The projected neighborhood of each Type I crossing, necessarily containing two Type IV crossings and one Type V crossing

Notice, as in Figure 1, that the neighborhood of each Type I crossing will also contain exactly two Type IV crossings and one Type V crossing, each of the same type (positive or negative). The same will also be true of Types II, VI, and VII, respectively.

We then obtain the following expression for aggregate slope, where $\mathbb{C}_{I}$ denotes the number of positive Type I crossings minus the number of negative ones, and similarly for Types II-IV, $\mathbb{C}_{\mathrm{II}}-\mathbb{C}_{\mathrm{IV}}$ represent the differences between the positive and negative crossings of the respective types.

$$
\begin{aligned}
l(S, L)=\frac{\mathbb{C}_{\mathrm{III}}+\mathbb{C}_{\mathrm{IV}}}{2}=\frac{\mathbb{C}_{\mathrm{III}}}{2}+\frac{2 \mathbb{C}_{\mathrm{I}}}{2} & =\frac{\mathbb{C}_{\mathrm{III}}}{2}+\left(\left(\mathbb{C}_{\mathrm{I}}+\mathbb{C}_{\mathrm{II}}\right)-2\left(\frac{\mathbb{C}_{\mathrm{II}}}{2}\right)\right) \\
& =\frac{\mathbb{C}_{\mathrm{III}}}{2}+(w(P)-2 \mathrm{k}(L)) .
\end{aligned}
$$

Rearranging gives

$$
\frac{\mathbb{C}_{\mathrm{III}}}{2}=l(S, L)-(w(P)-2 \operatorname{lk}(L))=\tau(S, P),
$$

as desired.

We also use this classification of the seven types of crossings in $L \cup \hat{L}$ to prove the following.

Proposition 2.2 Any spanning surface has an even aggregate slope.

Proof We proceed by induction on Euler characteristic, and assume $S$ to be connected, spanning $L$ (we can easily generalize to disconnected surfaces). If $\chi(S)=1, S$ is a disk, so aggregate slope is zero. Assume the proposition is true for $\chi(S)>-n$. Let $\chi(S)=-n$. Find an arc $\alpha$ on $S$ such that $S^{\prime}=S \backslash N(\alpha)$ is connected, spanning a link $L^{\prime}$. Since $\chi\left(S^{\prime}\right)=1-n$, it must have an even aggregate slope, by our inductive hypothesis. Now, isotope $S$ and $L$ to shorten $\alpha$, changing the projection to give the picture in Figure 2. Call the resulting projection of the link $P$. 


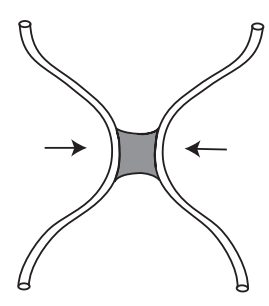

Figure 2: Shrinking $\alpha$

Cutting along $N(\alpha)$ will not create any new crossings, so any change in aggregate slope will result from positive crossings becoming negative crossings or vice versa, as a result of changes in orientation. Since $\hat{L}$ will inherit the orientation of $L$, all positive Type III crossings will remain positive, and all negative Type III crossings will remain negative. The only other crossings that contribute to aggregate slope are Type IV crossings, but each Type IV crossing will appear as in Figure 1, from which it follows that any change in the orientation of Type IV crossings will increase or decrease the aggregate slope by exactly two. Therefore, if $l\left(S^{\prime}, L^{\prime}\right)$ is even, then $l(S, L)$ will be as well, proving our statement.

Although writhe and linking number may vary with the orientation of a link, the slope, aggregate slope, and twist will not.

Proposition 2.3 Slope, aggregate slope, and linking number are invariant across all projections of isotopic embeddings of a given surface $S$ spanning a given oriented link $L$, but writhe and twist are not. Writhe and twist are invariant across all projections of isotopic embeddings of $S$ in which $L$ has a given orientation and is in reduced, alternating form.

Proof Isotopy of $S$ does not change the identity of the link $L \cup(S \cap \partial N(L))$, and since linking number is invariant across all projections, slope, aggregate slope, and linking number are invariant across all projected embeddings of $S$. Adding a half-twist to $P(L)$ alters the writhe, and the twist as well, since $\tau(S, P)=l(S, L)-(w(P)-2 \operatorname{lk}(L))$. Because writhe is invariant under flyping, it is invariant across all reduced, alternating projections of an oriented link $L$. Therefore, twist is as well.

Note that compression increases $\chi(S)$ by two, and boundary compression increases $\chi(S)$ by one.

Proposition 2.4 A meridianal boundary compression of a spanning surface yields another spanning surface. 
Proof Suppose that surface $S$ spans link $L$ and that $D$ is a meridianal boundarycompression disk. Then $\stackrel{\circ}{D} \cap S=\varnothing$ and $\partial D=\alpha \cup \beta$ where $\alpha \subset \partial N(\hat{L})$ for some link component $\hat{L}$ and $\beta \subset S \backslash N(\hat{L})$. Note that $\alpha$ intersects $S$ only at its endpoints, and that $S \cap \partial N(\hat{L})$ consists of two arcs sharing the endpoints of $\alpha$. We see then that we can isotope $D$ through $S^{3} \backslash S$, with its boundary moving across $S \cup \partial N(\hat{L})$, such that we obtain the general picture of a meridianal boundary compression shown in Figure 3 (in fact, there are two possible pictures of a meridianal boundary compression, the second being the reflection of the one shown in the figure).

Performing the boundary compression amounts to cutting $S$ along $N(D) \cap S$, and then gluing in two parallel copies of $D$. The picture shows that this yields a spanning surface, whose slope along $\hat{L}$ differs from that of $S$ by \pm 2 .

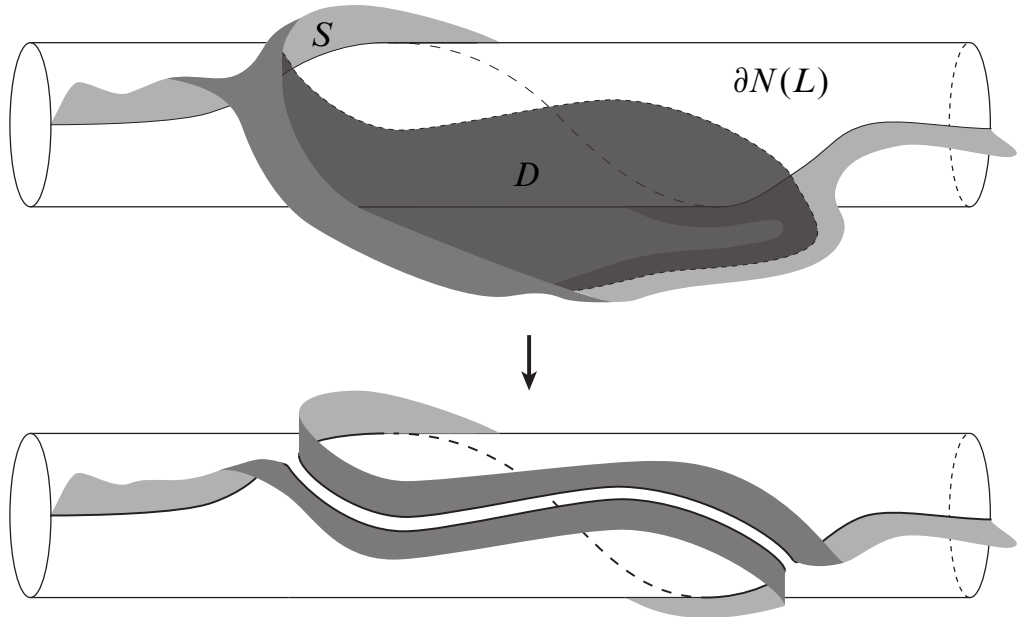

Figure 3: Meridianal boundary compression of a spanning surface yields another spanning surface.

Note that the meridianal boundary compression yields another spanning surface with slope that has increased or decreased by exactly two, while compression leaves the slope unchanged. Since neither compression nor meridianal boundary compression affects the writhe or linking number of an oriented projection, meridianal boundary compression increases or decreases twist by exactly two and compression leaves twist unchanged.

Seifert's algorithm for producing a Seifert surface for an arbitrary link need not generate a minimal genus Seifert surface, as the example in Figure 4 demonstrates. 


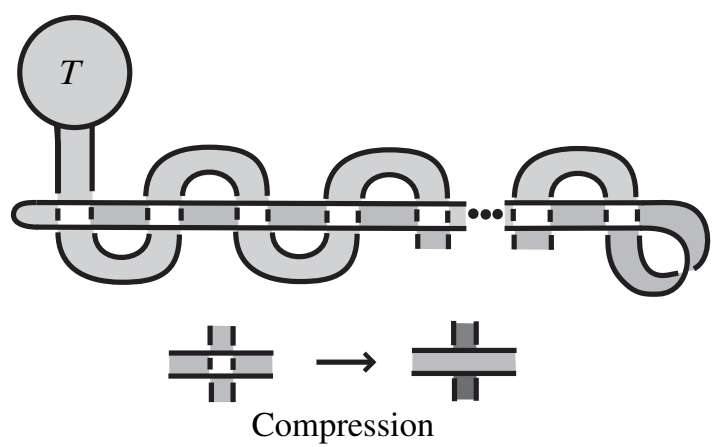

Figure 4: Seifert's algorithm can generate a surface that can be compressed an arbitrarily large number of times.

However, Theorem 1.1 shows that it does when the link is alternating. One would like to obtain a similar result in the case of nonorientable spanning surfaces, so it is worth considering Gabai's method of proof.

First, he shows that given orientable surfaces $S$ and $T$, each spanning an oriented link $L$, with $g(T)<g(S)$, there exists an orientable surface $T^{\prime}$ with smaller genus than $S$ such that $\stackrel{\circ}{T}^{\prime} \cap \stackrel{\circ}{S}=\varnothing$. Second, he takes a surface $S$ obtained by applying Seifert's algorithm to an $n$-crossing oriented link and assumes there exists an orientable spanning surface of smaller genus. He then uses the earlier result to construct a surface $T$ of smaller genus than $S$, where the two are disjoint in their interiors. He then uses this structure to find arcs on $S$ and $T$ that are parallel to the same crossing, and he cuts along each. This reduces by one the crossing number of their shared boundary and increases by one each surface's Euler characteristic. He now has surfaces $S^{\prime}$ and $T^{\prime}$ spanning an $(n-1)$-crossing alternating link, where $S^{\prime}$ comes from Seifert's algorithm and $g\left(T^{\prime}\right)>g\left(S^{\prime}\right)$. From here, he applies an inductive argument on crossing number and reaches a contradiction.

The proof is elegantly elementary but fails to generalize to nonorientable surfaces because of the following.

Proposition 2.5 No two spanning surfaces of the same link, at least one of which is nonorientable, can be disjoint in their interiors.

Proof Given two surfaces disjoint in their interiors, spanning the same link, they must have the same boundary slopes. We can then connect their two boundaries with an annulus in $\partial N(L)$ to obtain a single, closed surface without boundary, embedded in 
3-space. If either spanning surface is nonorientable, then the closed surface will be as well. Since no closed, nonorientable surface can be embedded in 3-space, we have a contradiction.

Before discussing nonorientability further, we present one more useful fact. Suppose a surface $S$ spans an oriented link $L$. For any $\operatorname{arc} \alpha \subset S$, with both endpoints on $L$, $N(\alpha) \cap S$ will take one of the forms pictured in Figure 5. We will call the former a deorienting band and the latter an orienting band.

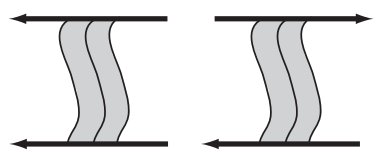

Figure 5: A deorienting band and an orienting band

Proposition 2.6 A surface $S$ spanning $L$ is orientable if and only if it is possible to orient $L$ so that $S$ contains no deorienting bands. Equivalently, $S$ is nonorientable if and only if $S$ contains a deorienting band under any orientation of $L$.

Proof If a surface is orientable, its boundary inherits an orientation from an orientation of the entire surface. The existence of a deorienting band would contradict the orientation inherited by the boundary. Conversely, if the surface is nonorientable it contains a Mobius band. Let $\beta \subset S$ be the core curve of this Mobius band and let $\gamma_{1}$, $\gamma_{2} \subset S$ be arcs with one endpoint on $\beta$ and the other on $L$. The endpoints of $\gamma_{1}$ and $\gamma_{2}$ on $\beta$ divide it in two. Let $\beta_{1}$ and $\beta_{2}$ be these two arcs of $\beta$, as in Figure 6.

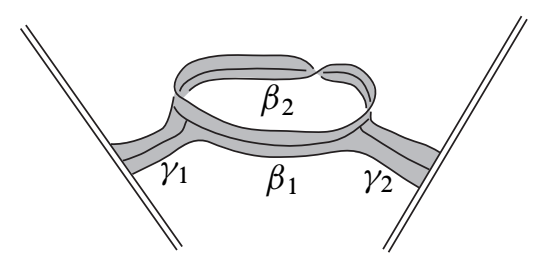

Figure 6: $N\left(\gamma_{1} \cup \beta \cup \gamma_{2}\right) \cap S$

Then under any orientation of $L$ either $N\left(\gamma_{1} \cup \beta_{1} \cup \gamma_{2}\right)$ or $N\left(\gamma_{1} \cup \beta_{2} \cup \gamma_{2}\right)$ will be a deorienting band. 
This will be an important fact in the proof of Theorem 5.4. As for the matter at hand, we say that a spanning surface $S$ is orientable relative to its oriented boundary link $L$ if $L$ is oriented in the way guaranteed by the above result. Note that any orientable surface $S$ can be oriented (assigned a normal direction) in two ways, and each of these orientations defines an orientation on its link boundary, $L$. These orientations of $L$ are precisely the ones relative to which $S$ is oriented. The example in Figure 7 may be useful in seeing how this distinction is important for Theorem 5.4, since this surface is orientable (it contains no Mobius band) but not relative to its oriented boundary (it contains deorienting bands).

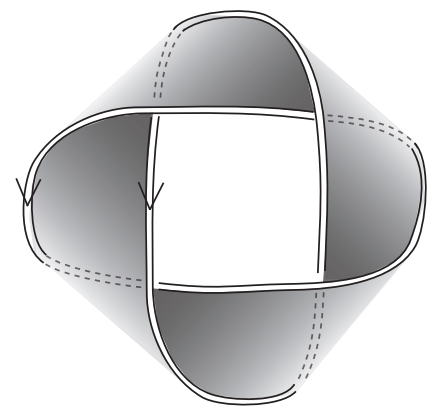

Figure 7: This annulus spanning the $4_{1}^{2}$ link is orientable because it contains no Mobius band, but not with respect to its oriented boundary, since it contains a deorienting band.

\section{State surfaces}

\subsection{Construction}

In Adams [3] and Futer, Kalfagianni and Purcell [12], checkerboard surfaces generated from reduced, alternating projections were shown to be essential, nonaccidental, and nonfibered and are therefore quasi-Fuchsian surfaces in hyperbolic link complements. We seek to describe a larger class of essential, nonaccidental, nonorientable surfaces spanning these links. Since fibers must be orientable, any such surfaces must be quasiFuchsian. To this end, we devised the following natural extension of Seifert's algorithm, as depicted in Figure 8.

State surface algorithm (1) Beginning with an alternating projection $P(L)$, split open each crossing in one of the two directions. 
(2) This will result in a collection of nonoverlapping, possibly nested circles. Choose a region on the projection sphere to contain $\infty$. Put nested circles at different heights relative to $S^{2}$ and fill each circle with a disk to the side that does not contain $\infty$ when projected to $S^{2}$.

(3) Connect the disks with half-twist bands at the crossings, yielding a surface with boundary equal to the link such that the link projects to $P(L)$, as in Seifert's algorithm, if it is possible to do so without a crossing band intersecting an existing disk. If this is not possible, change the heights of the disks so that the disks can be connected with crossing bands.

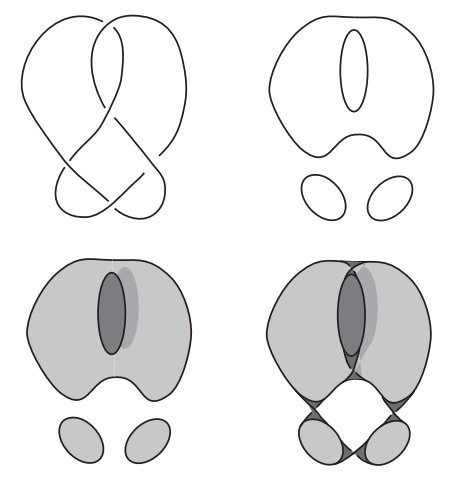

Figure 8: Constructing a state surface

Not all choices of relative disk heights can generate a state surface, as the crossing band between two disks might intersect a third disk. It is always possible, though, to choose heights for the disks to generate a state surface, most simply by stacking all inner disks (disks with boundary circles inside boundary circles of other disks) above outer ones. Note that while different choices of relative heights for nested circles may well generate distinct state surfaces, the choices of disk heights will not affect Euler characteristic, orientability, or aggregate slope.

State surfaces that have a crossing band that connects a circle to itself are often boundarycompressible (in fact, meridianally boundary-compressible). To avoid this issue, we define a basic state surface to be one that does not have a crossing band connecting a circle to itself. For our purposes, the only state surfaces with which we will be concerned are basic state surfaces. In a subsequent paper, we plan to discuss the questions of essentiality and nonaccidentality of these surfaces. 


\subsection{Properties}

In 1991, W Menasco and M Thistlethwaite [23] (also [25]) used Menasco's geometric structure to prove Tait's Flyping Conjecture which states that any two reduced, alternating projections of the same link are related through a finite sequence of flypes. Let us consider what happens to state surfaces under flyping.

Proposition 3.1 Given a state surface $S$ generated from an alternating projection $P$ and a flype that takes $P$ to projection $P^{\prime}$, there exists a state surface $S^{\prime}$ obtained from $P^{\prime}$ with the same genus, orientability, and aggregate slope as $S$, where $S^{\prime}$ is a basic state surface if and only if $S$ is.
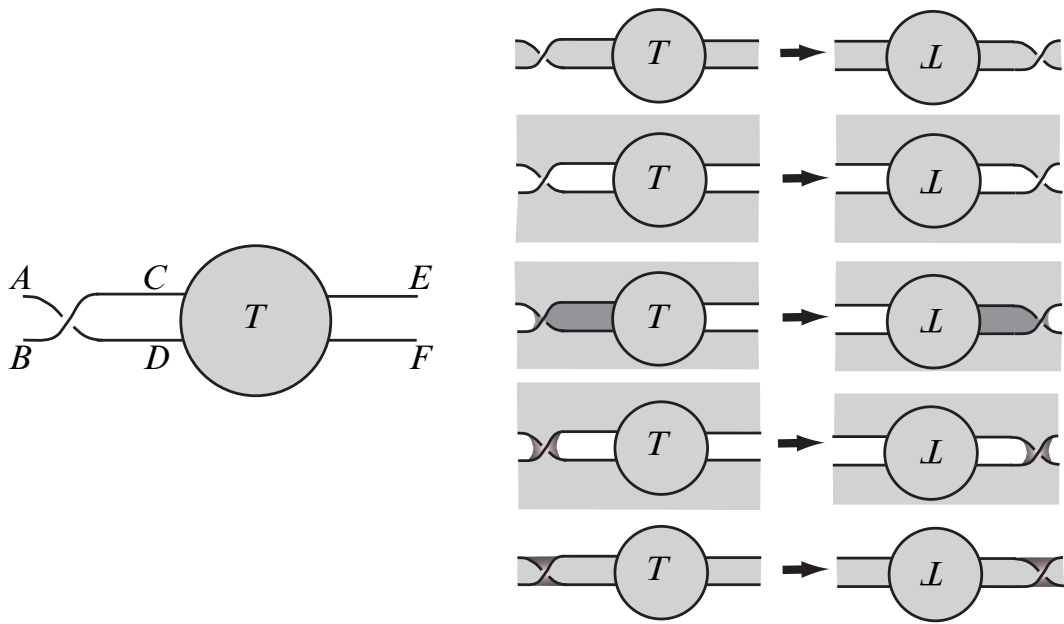

Figure 9: The five possible appearances of a state surface relative to a flype, together with the state surface for the resulting projection that has the same genus, orientability, and aggregate slope as the original

Proof See Figure 9. We have eight possible situations, depending on the crossing splits. After splitting, there are two possibilities for the single crossing; it can be split vertically or horizontally. After splitting in $T$, there are two possibilities. It could be the case that $C$ shares a circle with $D$ and $E$ shares a circle with $F$. Or it could be that $C$ shares a circle with $E$ and $D$ shares a circle with $F$. Finally, there are two possibilities for the splittings outside this part of the projection. Either $A$ is connected to $E$ and $B$ to $F$, or $A$ is connected to $B$ and $E$ to $F$. Three of the eight possibilities yield figures equivalent to three others, yielding five figures to consider. For each, we 
perform a flype about the tangle $T$. Since $S$ is state, $S$ is a state surface both to the inside and outside of $T$. In each case, we can obtain a surface after the flype with the same genus, orientability and aggregate slope as the original surface. Note that the original and resulting surface are either both basic or neither basic. Also note that the last two cases are never basic state surfaces and always nonorientable.

So any two alternating projections of the same link generate equivalent collections of state surfaces, up to genus, orientability and aggregate slope.

For our next fact, notice that in the construction of a state surface we have two options in the splitting of each crossing, which we will call $A$ and $B$ splits, as in Figure 10. As is standard (cf Adams [1, Chapter 6]), the regions adjacent to a crossing can be labelled $A$ and $B$ depending on whether we twist the top strand counterclockwise or clockwise to cover those regions. The $A$ or $B$-split corresponds to creating a channel between the two regions with that label at the crossing.
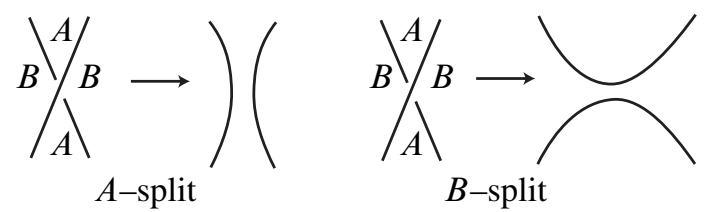

Figure 10: $A$ and $B$ splittings in the construction of a state surface

Proposition 3.2 Suppose that the construction of a state surface $S$ involves $a A$-splits and $b \quad B$-splits. Then $\tau(S, P)=a-b$. It then follows that

$$
l(S, L)=a-b+w(P)-2 \operatorname{lk}(L) .
$$

Proof Recall that $\tau(S, P)$ can be calculated just by counting the net total of positive and negative crossings between $L$ and $S \cap \partial N(L)$ through annuli in $S \cap N(L)$. If $S$ is state, we may have some pairs of crossings that appear as in Figure 11, but each pair will have no net effect on $\tau(S, P)$.

Since $S$ is state, the only other crossings between $L$ and $S \cap(\partial N(L))$ through annuli in $S \cap N(L)$ will occur at crossings (exactly why this is true will become more apparent when we introduce the Menasco structure, although it should seem believable from our pictures so far). The neighborhood of each $A$-split crossing in $P(L)$ will contain two positive crossings between $L$ and $S \cap(\partial N(L))$ through an annulus in $S \cap N(L)$, and the neighborhood of each $B$-split crossing will contain two negative crossings of this type. Thus, $\tau=(2 a-2 b) / 2=a-b$. That $l(S, L)=a-b+w(P)-2 \operatorname{lk}(L)$ then follows from the definition of $\tau(S, P)$. 


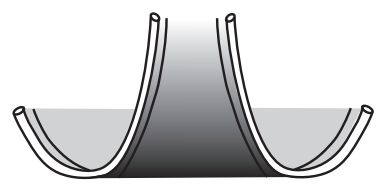

Figure 11: The positive and negative crossings between $L$ and the parallel curve on $S$ cancel each other out wherever $S$ takes this form.

Note that a state surface constructed from an $n$-crossing projection in which the crossing splits produce $f$ circles will have an Euler characteristic of $f-n$.

Suppose we want to find a minimal genus state surface for a given projection. We could compare the genera of all $2^{n}$ possible surfaces, but we would like to do better than this. Instead, we can employ the following.

Minimal genus algorithm (1) Find the smallest $m$ for which the projection contains a projection $m$-gon.

(2a) If $m \leq 2$, choose a projection $m$-gon and split each crossing on this region's boundary so that this region becomes a circle.

(2b) If $m>2$ (and therefore $m=3$, by a simple Euler characteristic argument on the projection plane), choose a projection 3-gon. From here, create two branches of our algorithm, each of which will ultimately yield a state surface. We will later choose to follow the branch that produced the smaller genus surface and to ignore the other. For one of these branches, we split each crossing on this projection 3-gon's boundary so that it becomes a circle. For the other branch, we split each of these three crossings the opposite way.

(3) Repeat until each branch reaches a projection without crossings. Of all constructed surfaces, choose the one with the smallest genus.

Theorem 3.3 The minimal genus algorithm always generates a minimal genus state surface spanning a given alternating link.

Note, however, that the resulting surface is not necessarily uniquely determined by this algorithm.

Proof (By induction on crossings in $P$.) The statement is trivial for zero crossings. Suppose it is true for fewer than $n$ crossings, and let $P$ be an $n$-crossing projection. 
Suppose that performing this algorithm on $P$ generates a state surface $S$ spanning $L$. Also, let $T$ be a minimal genus state surface spanning $L$; then $g(T) \leq g(U)$ for any state surface $U$ spanning $L$. We want to show that $g(S) \leq g(T)$, or equivalently that $f(S) \geq f(T)$, where $f$ is the number of circles used in the construction of each respective state surface.

First suppose that $P$ contains a projection 1 -gon or 2 -gon. Let $G$ be the one around which all crossings are split in the construction of $S$, via our algorithm. Also, suppose for contradiction that $f(S)<f(T)$. We construct a new state surface, $T^{\prime}$, with the exact same crossing splits as in the construction of $T$, except that the crossing splits around $G$ coincide with the corresponding crossing splits for $S$. Note that if the crossing splits of $S$ and $T$ already coincide here, we will have $T=T^{\prime}$. Figure 12 demonstrates that $f\left(T^{\prime}\right) \geq f(T)$. Note that the first, third and fourth cases depicted cause a decrease in the number of circles if the crossing splits are switched, while in the second case, the number of circles may either decrease or be preserved.

We then have an $(n-1)$ or $(n-2)$-crossing projection where our algorithm yields $f(S)$ circles and where the construction of $T^{\prime}$, another state surface, yields a projection of $f\left(T^{\prime}\right) \geq f(T)>f(S)$ circles. This contradicts our inductive hypothesis.

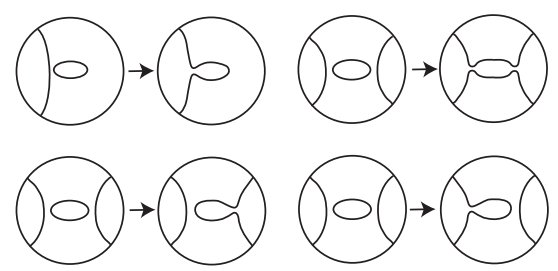

Figure 12: Changing the crossing splits around a split 1-gon or split 2-gon in our algorithm cannot increase the number of circles.

Assume then that $P$ contains no projection 1 -gons or 2 -gons. Let $G$ be the projection 3-gon around which we first split crossings in the construction of $S$. Let $P_{1}$ be the projection obtained from $P$ by splitting the crossings around $G$ so that $G$ becomes a circle. Let $P_{2}$ be the projection obtained from $P$ by splitting each of these three crossings the opposite way. $P_{1}$ and $P_{2}$ both have $n-3$ crossings. Let $S_{1}$ and $S_{2}$ be the state surfaces our algorithm generates from these respective projections. Note that $f(S)=\max \left\{f\left(S_{1}\right), f\left(S_{2}\right)\right\}$. Let $T_{1}$ and $T_{2}$ be state surfaces generated from $P_{1}$ and $P_{2}$, respectively, with crossing splits agreeing with those of $T$. Our inductive hypothesis implies that $f\left(S_{1}\right) \geq f\left(T_{1}\right)$ and $f\left(S_{2}\right) \geq f\left(T_{2}\right)$. Figure 13 shows that either $f\left(T_{1}\right) \geq f(T)$ or $f\left(T_{2}\right) \geq f(T)$. It follows that $f(S) \geq f(T)$. 


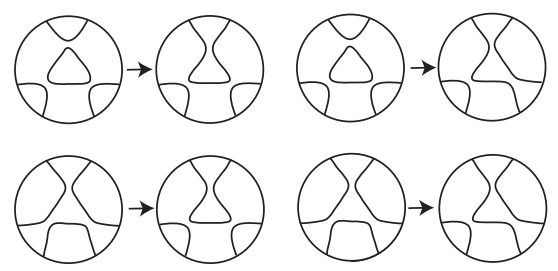

Figure 13: Changing the crossing splits around a split 3-gon from our algorithm cannot increase the number of circles for both possible initial splittings.

Therefore our algorithm generates a minimal genus state surface. Theorem 5.4 will imply that this surface is in fact a minimal genus spanning surface (state or nonstate) for our link.

For Theorem 5.4, we extend the class of allowed surfaces in a manner that increases genus and perhaps changes boundary slope. To do this, we allow the addition of crosscaps and handles to a basic state surface. These additions can be thought of as infinitesimal, local processes that take place in some isolated, unimportant (away from the crossings) part of the surface along the boundary. These processes are depicted in Figure 14.
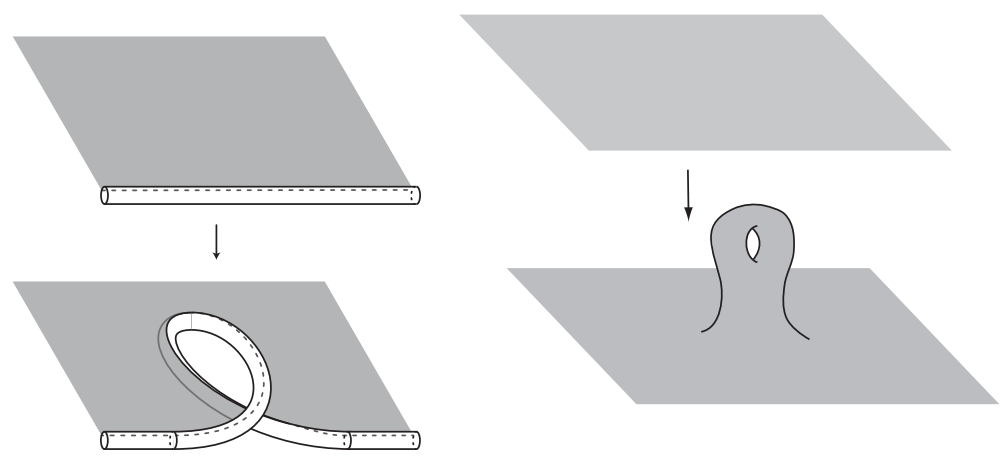

Figure 14: Adding a crossscap (left) and adding a handle (right)

Neither adding a crosscap nor adding a handle need alter the projection $P(L)$. We can see this in the picture above for the handle, and in the later picture (see Figure 26) of adding crosscaps to a surface in Menasco form. Adding a crosscap will either increase or decrease the twist (and therefore the aggregate slope) by two and will decrease the Euler characteristic by one. Adding a handle will decrease the Euler characteristic by 
two and will not change twist or slope. Adding a crosscap or a handle will necessarily produce an inessential surface.

Note that a minimal nonorientable genus spanning surface need not be essential. It can be the case that the minimal genus algorithm generates only orientable surfaces, in which case the minimal nonorientable genus surface is obtained from one of these by adding a crosscap, and the nonorientable genus is $\frac{1}{2}$ greater than the orientable genus. (The knot $7_{4}$ is an example of this.) But this is as much greater than the orientable genus as the nonorientable genus can ever be.

We are now ready to develop the primary piece of machinery that we use toward the proof of Theorem 5.4.

\section{Menasco structure}

We utilize William Menasco's geometric machinery for analyzing alternating links as first appeared in Menasco [21]. A variety of results have been proved using this technique, including the fact that any two reduced alternating projections of a given link are related through flyping (Menasco and Thistlethwaite [22] and [25]), an alternating link is splittable if and only if its alternating projections are disconnected, and an alternating knot is composite if and only if it is obviously composite in any reduced alternating projection [21]. Additional results for alternating links and extensions of alternating links have also been proved (eg Adams, Brock, Bugbee, Comar, Faigin, Joseph and Pesikoff [4]; Adams [2]; Adams, Dorman, Foley, Kravis and Payne [5]; Adams, Fleming, Levin and Turner [6]; Hayashi [16]; Menasco et al [22]; Menasco and Thistlethwaite [24] and Tsukamoto [36]). We will use the Menasco machinery to prove that a spanning surface must have an arc whose neighborhood in $S$ can be isotoped into a "crossing band". This will allow an inductive argument to follow.

\subsection{Background}

Begin with a reduced, alternating projection on a sphere. From this projection, create an embedding of $L$ that lies on a sphere, $S^{2} \subset S^{3}$, except in the neighborhood of each crossing, where we insert a ball so that the overstrand can run over this ball and the understrand can run under it. We will call every such crossing ball a Menasco ball. This will give rise to a picture as in Figure 15 on the left and a view from above as on the right. We will assume that $L$ is not splittable. Otherwise, it would appear obviously so in the projection (see Menasco [21]) and we could consider each nonsplittable component separately. 

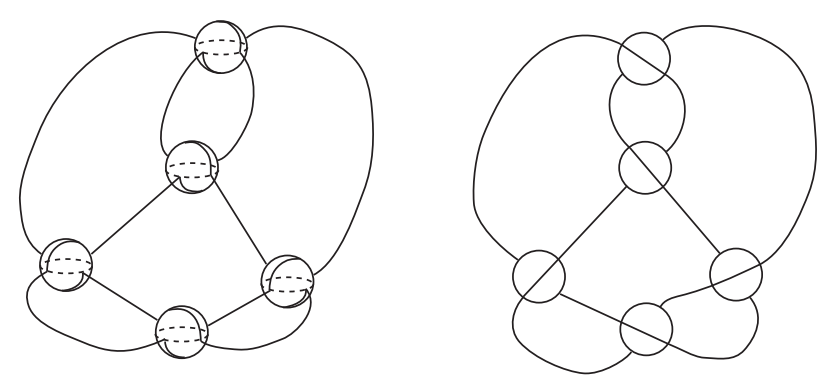

Figure 15: A embedding of the $5_{2}$ link in Menasco form

We will call such an embedding of $L$ relative to $S^{2}$ a Menasco projection $P$ with $n$ crossings. We denote the union of the collection of Menasco balls by $M=\bigcup_{i=1}^{n} M_{i}$ and we let $F=S^{2} \backslash \stackrel{\circ}{M}$.

Now suppose we have an incompressible (but not necessarily boundary-incompressible) surface $S$ spanning $L$. By general position, we can isotope $S$ so that $S \cap F$ is a collection of simple closed curves and arcs with each endpoint either on $L$ or on the equator of a Menasco ball. We can also choose this isotopy so that $S$ is disjoint from the interiors of the Menasco balls, wherever possible, including everywhere along $N(L)$. That is, $S \cap N(L) \cap M=\varnothing$. Note that later, we will qualify this requirement to allow intersections of $S$ with $N(L) \cap M$ called "crossing bands".

We assume that $N(L)$ is small relative to the Menasco balls. Utilizing the incompressibility of $S$, we can isotope $S$ to eliminate all simple closed curves in $S \cap F$.

Menasco shows that once we isotope $S$ in this way, anywhere it is still forced to intersect the interior of a Menasco ball it must do so in a saddle as in Figure 16.

We define an intersection arc to be an arc of $S \cap F$, with each endpoint lying either on the link or on the equator of a Menasco ball. Each intersection arc lies entirely within a single connected region in $F \backslash P$.

Then, for each Menasco ball $M_{i}$, each of the four regions of $F \backslash P$ adjacent to $M_{i}$ will have the same number of endpoints of intersection arcs on $\partial M_{i}$, that number being the number of saddles. Define $F_{+}$to be the union of $F$ with the upper hemispheres of the Menasco balls and $F_{-}$to be the union of $F$ with the lower hemispheres. Then at a Menasco ball with a single saddle, the intersection curves of $S$ with $F_{+}$and $F_{-}$ appear as in Figure 17.

Since $S$ is incompressible, we then see that $F \cup \partial M$ cuts $S$ up into a collection of disks, some inside the sphere, some outside the sphere, and some inside Menasco balls. 


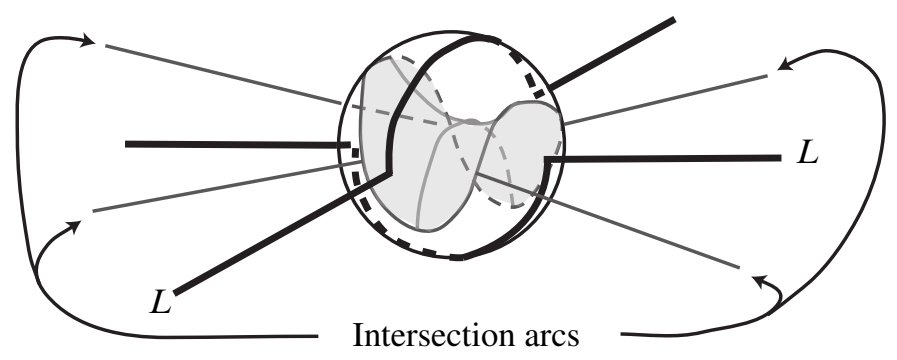

Figure 16: The surface intersects each Menasco ball in a possibly empty collection of saddles.

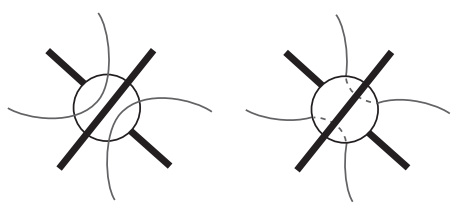

Figure 17: Pictures of the arcs of intersection between $S$ and $F_{+}$, and between $S$ and $F_{-}$

We will call these underdisks, overdisks, and saddles, respectively. Up to isotopy, $S$ is then uniquely determined by the way it intersects $F$. A final important fact from Menasco [21] is that no intersection arc has both endpoints on $\partial M_{i}$ for any Menasco ball $M_{i}$. Otherwise, it could easily be removed.

We call an incompressible surface that has been isotoped in this manner to be in Menasco form. Figure 18 depicts a state surface for the $5_{2}$ knot that has been isotoped to be in Menasco form. We see $F_{+}$with the overdisks shaded and the underdisks labelled $U_{i}$. Note that all state surfaces can be isotoped to be in Menasco form without any saddles.

\subsection{Cleaning}

In this section, we describe some means to simplify surfaces in Menasco form. In general, we want to minimize the number of intersection arcs between $S$ and $F$, so several of our results will describe ways of doing this. This will generally involve isotoping $S$ or altering it in some other well-defined way to eliminate particular types of arcs of intersection, or else showing that certain types of arcs of intersection 


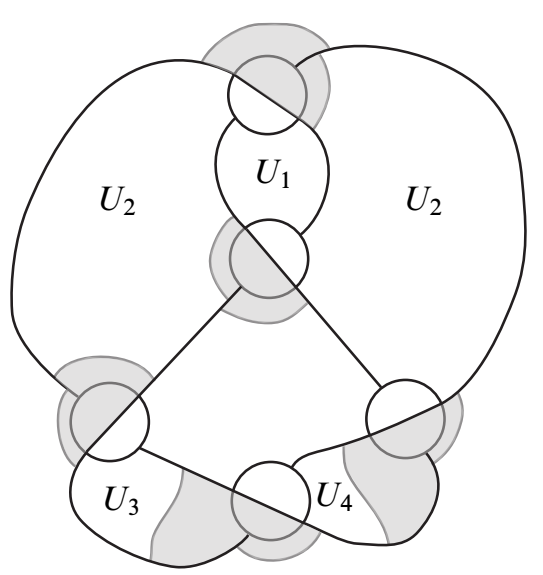

Figure 18: The view of $F_{+}$depicting a state surface spanning the $5_{2}$ knot, isotoped to lie in Menasco form, with overdisks shaded and underdisks labelled

simply cannot occur. As before, we call our spanning surface $S$, our link $L$, our Menasco projection $P$ with $n$ crossings, our projection surface $S^{2}$, and our collection of Menasco balls $M$. Also, as before, we let $F=S^{2} \backslash \stackrel{\circ}{ }$ be our projection surface.

Given an intersection arc, there exists an overdisk and an underdisk, each of which contains that arc on its boundary. Additionally, each point on $L$ lies on the boundary of either an overdisk or an underdisk, with each intersection arc endpoint on $L$ marking the transition between the boundary of an overdisk and the boundary of an underdisk. Each intersection arc endpoint on a Menasco ball boundary also lies on the boundary of a saddle. Every point on the boundary of an overdisk or underdisk lies on an intersection arc, on the boundary of a Menasco ball, or on $L$. For the proof of Theorem 5.4, we heavily utilize this alternation between overdisks and underdisks.

In accordance with this alternation of overdisk and underdisk boundaries on $L$, where "+" denotes a portion of the boundary of an overdisk and "-" denotes a portion of the boundary of an underdisk, and transitions occurring exactly at the endpoints of intersection arcs, we can see that every intersection arc with endpoints on $L$ will take one of the forms shown in Figure 19.

If we imagine cutting $S$ along any of the four types of arc, by removing a neighborhood of this arc from $S$, a new surface $S^{\prime}$ is generated, bounding a new link $L^{\prime}$, which will inherit a projection $P^{\prime}$. If we cut along either of the first two types of $\operatorname{arcs}, P^{\prime}$ will still be alternating with $n$ crossings. We call these first two flat intersection arcs. If we cut along either of the latter two types of arcs, $P^{\prime}$ will have $n+1$ crossings. For one of 


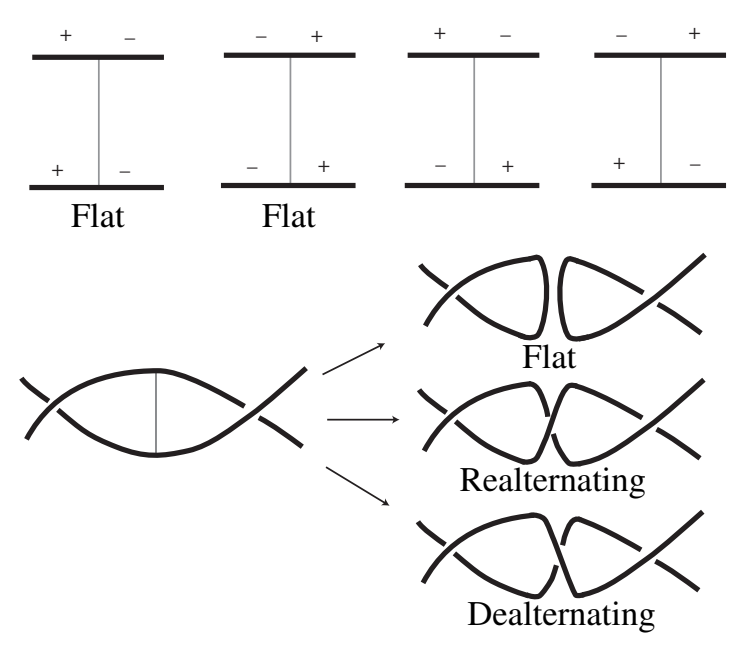

Figure 19: Four possibilities for intersection arcs and the possible results from cutting along an arc

these cases, $P^{\prime}$ will no longer be alternating, while for the other it will be. We will call the former arc a dealternating intersection arc and the latter a realternating intersection arc. Every intersection arc with both its endpoints on $L$ will be flat, dealternating, or realternating, as in Figure 19.

Among all embeddings of $S$ relative to $F \cup M$ that preserve the already established structure (Menasco projection, general position, $S$ disjoint from $M$ wherever possible), we want to choose the best one. In particular, we want to lexicographically minimize the number of intersection arcs and saddledisks. That is, we want to minimize the number of intersection arcs, and once we have done this we want to minimize the number of saddledisks as well. If an embedding of $S$ relative to $F \cup M$ does this, we will say that it is clean.

Proposition 4.1 In a clean embedding, no adjacent pair of intersection arc endpoints on $P \cap F$ will connect to intersection arcs that both lie in the same region of $F \backslash P$, as in Figure 20.

Proof Suppose our clean embedding contains such a pair of adjacent intersection arcs, as in 20. The portion of $P \cap F$ connecting the two intersection arc endpoints lies on the boundary of either an overdisk or an underdisk. We can isotope this disk through $F$ along the portion of $P \cap F$ that connects the two intersection arc endpoints. Doing this will decrease the number of intersection arcs, contradicting the fact that our Menasco structure was clean. 


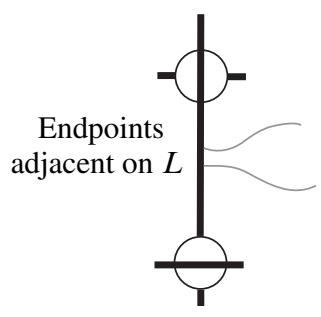

Figure 20: No clean embedding of a surface in Menasco form contains this conformation.

Proposition 4.2 Suppose $D$ is an overdisk (underdisk) whose boundary contains intersection arcs $I$ and $J$, both in some region $R \subset F \backslash P$ where $I$ and $J$ can be connected by an arc $\alpha \subset F \backslash(P \cup S)$. Then, we can push a particular part of $D$ through $S^{2} \cap N(\alpha)$ so that it is divided into two separate overdisks (underdisks). If our original embedding of $S$ was clean, then this will be as well.
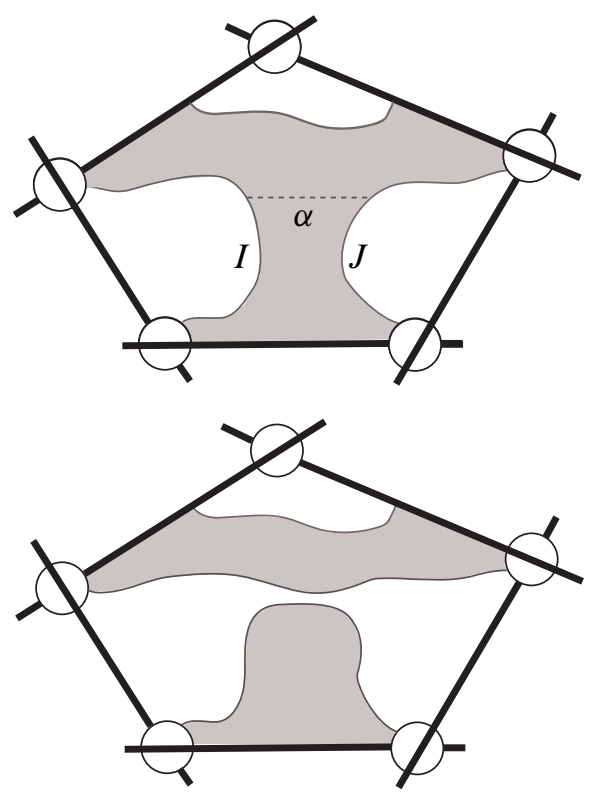

Figure 21: Pushing the disk $D$ through the projection plane

Proof See Figure 21. Suppose we have such a $D, R, I, J$, and $\alpha$. Let $\beta \subset D$ be an arc with the same endpoints as $\alpha$. Then $\alpha \cup \beta$ bounds a disk $X$ such that

$$
N(\partial X) \cap S \cap \stackrel{\circ}{X}=\varnothing
$$


and $L \cap \stackrel{\circ}{X}=\varnothing$. Therefore $\stackrel{\circ}{X} \cap S$ will consist entirely of simple, closed curves of intersection, each of which can be removed because $S$ is incompressible. Therefore, we can assume that $\stackrel{\circ}{X} \cap S=\varnothing$. We can then isotope $N(\beta)$ through $S^{2} \cap N(\alpha)$ via $N(X)$, so that $D$ is divided into two separate overdisks, as desired.

Since the number of intersection arc endpoints does not change, the number of intersection arcs will not change. Also, $S$ was originally in Menasco form, and we have done nothing to change this. If the original embedding was clean, this will be as well.

Note that this move is not a simplification, but rather a convenient freedom. This convenience will soon become manifest.

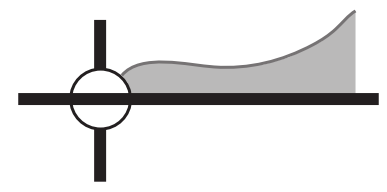

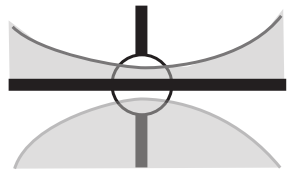

Over

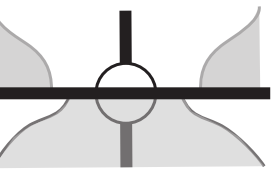

Over

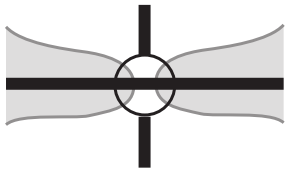

Under
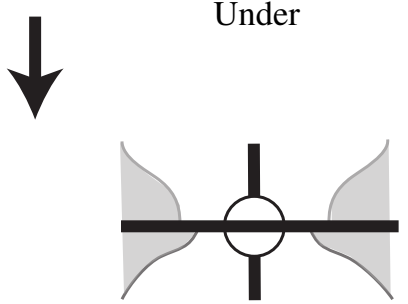

Under

Figure 22: Another removable structure in our cleaning process

Proposition 4.3 Suppose $S$ is in a clean embedding, and $D$ is either an overdisk or an underdisk whose boundary contains intersection arc $I$ in some region $R \subset F \backslash P$ where an endpoint of $I$ lies on a Menasco ball $M_{i}$. Then $\partial D$ will not contain either portion of $L$ that traverses $M_{i}$, as in the top of Figure 22.

Proof Suppose, without loss of generality, that $D$ is an overdisk. Clearly $\partial D$ will not contain the underpass of $M_{i}$. Suppose it contains the overpass. The bottom of Figure 22 shows the isotopy that removes the corresponding saddledisk at $M_{i}$ without 
increasing the number of intersection arcs, contradicting the fact that our embedding of $S$ was clean.

The next two lemmas assume that the spanning surface in question is also meridianally boundary-incompressible.

Proposition 4.4 If $S$ is meridianally boundary-incompressible in a clean embedding, then no intersection arc has both endpoints on the same component of $P \cap F$.

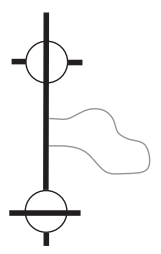

Figure 23: An additional removable structure in our cleaning process

Proof Suppose we have such an intersection arc, $I$. See the two conformations in Figure 23. The region of $F$ bounded by $I$ together with the portion of $P \cap F$ that connects the endpoints of $I$ may or may not contain additional arcs of intersection in its interior. If it does, choose an innermost one, $I^{*}$. Now the region of $F$ bounded by $I^{*}$ together with the portion of $P \cap F$ that connects the endpoints of $I^{*}$ does not intersect $S$ in its interior. This region of $F$ is a disk whose boundary lies entirely on $P$, except along $I^{*} \subset S$ and that does not intersect $S$ in its interior. Therefore, either this disk represents a meridianal boundary compression or $I^{*}$ cuts a disk off of $S$ and can thus be pushed into $L$, reducing the number of intersection arcs. The former possibility contradicts our assumption that $S$ is meridianally boundary-incompressible, and the latter contradicts the assumption that our embedding of $S$ is clean. Therefore, no such $I$ can exist.

Proposition 4.5 Suppose $S$ is meridianally boundary-incompressible. A clean embedding will contain no intersection arc $I$ with one endpoint on a Menasco ball boundary $\partial M_{i}$ and the other on a portion of $P \cap F$ that connects to $M_{i}$, as in Figure 24.

Proof Suppose we do have such an intersection arc $I$. Consider the region bounded by $I, \partial M_{i}$, and the portion of $P \cap F$ that contains an endpoint of $I$. Notice that any intersection arc in this region must have one endpoint on $\partial M_{i}$ and the other on $P$, since no intersection arc can have both endpoints on either of these, as shown earlier 


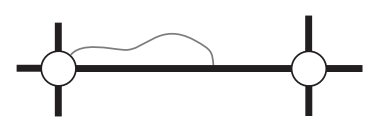

Figure 24: A final conformation for our cleaning process to remove

by Menasco and by Proposition 4.4. Choose an innermost arc of intersection; that is, one whose analogously bounded region does not intersect $S$ on its interior. Call this intersection $\operatorname{arc} I^{*}$.

Now consider the portion of $P \cap F$ connecting the endpoint of $I^{*}$ on $P$ with $\partial M_{i}$. Its interior will either contain no intersection arc endpoints or exactly one endpoint, that being of an intersection arc that lies in the region of $F \backslash P$ adjacent to the one containing $I$ and $I^{*}$.

First suppose that this portion of $P$ contains no intersection arc endpoints. Then, we get an immediate contradiction by Proposition 4.3.
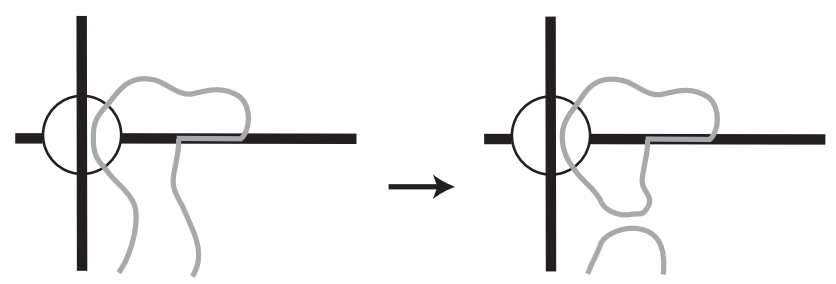

Figure 25: Using Proposition 4.2 to obtain a potential boundary-compression disk

Now suppose that the aforementioned portion of $P$ contains one intersection arc endpoint. This gives the picture in Figure 25, which Proposition 4.2 allows us to alter as shown. We then have a disk (depicted here as an overdisk) whose boundary lies entirely on the interior of $S$, except for a single arc along $P$. Then, either this disk represents a meridianal boundary compression (contradicting the fact that $S$ is meridianally boundary-incompressible) or its boundary cuts a disk off of $S$ and can therefore be pushed into $L$, which would decrease the number of intersection arcs, thereby contradicting the assumption that $S$ was embedded cleanly.

Also, now that we have the Menasco structure at our disposal, we can provide a different picture for what it means to add a crosscap. One can add a crosscap to a surface in Menasco form by performing one of the two operations shown in Figure 26. This has the same impact as adding a crosscap as in Figure 14 and then pulling the knot taut. 


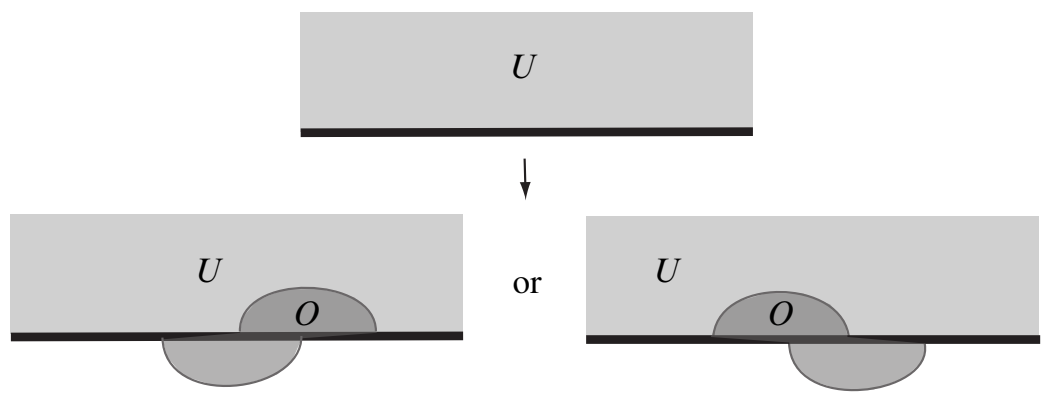

Figure 26: Adding a crosscap to a surface in Menasco form corresponds to adding two intersection arcs as in this figure.

\subsection{Combinatorial properties}

Suppose that we have a clean embedding of a meridianally essential surface $S$. In this section, we will lay out several combinatorial properties of $S \cap F$. From these, the proof of Theorem 5.4 will follow somewhat naturally.

Suppose we incorporate into a Menasco projection $P$ a collection $A=\bigcup_{i=1}^{r} A_{i}$ of realternating intersection arcs, all of whose endpoints lie on $P$ (rather than on a Menasco ball). Recall that because $S$ is meridianally boundary-incompressible none of these will have both endpoints on the same edge of a projection $n$-gon of $P$. We define a segment of $P$ with respect to $A$ to be a portion of $P \cap F$ that is disjoint on its interior from endpoints of arcs in $A$ and each of whose endpoints lies either on the boundary of a Menasco ball $M_{i}$ or at an endpoint of an arc in $A$. See Figure 27. Note that if $A=\varnothing$ then each segment connects a pair of adjacent Menasco balls.

Let $P^{*}=F \cap(P \cup A)$. As each segment lies on the boundaries of exactly two regions of $F \backslash P^{*}$, we can associate two "boundary segments" to any segment. We thus define a boundary segment of $P$ with respect to $A$ to be a pairing of a region $R$ of $F \backslash P^{*}$ with a segment of $P$ (with respect to $A$ ) on its boundary. As with a segment, given a boundary segment that lies on the boundary of a particular region, each endpoint of that boundary segment either will be an intersection arc endpoint on that region's boundary or will lie on the boundary of a Menasco ball that forms a portion of that region's boundary.

For the following remarks, suppose that our projection $P$ contains $n$ crossings, that $A$ contains $r$ realternating arcs, all of whose endpoints lie on $P$, and that $P^{*}=$ $F \cap(P \cup A)$, as before. 


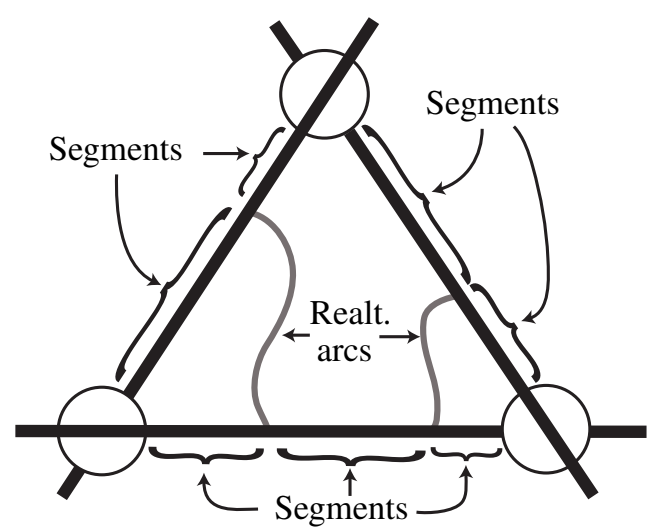

Figure 27: Segments of $P$

Proposition 4.6 $P^{*}$ will contain exactly $2 n+2 r$ segments and $4 n+4 r$ boundary segments. Each segment will lie on the boundary of an overdisk at one end and the boundary of an underdisk at the other.

Proof First suppose that $r=0$; that is, $A=\varnothing$. Each of the $n$ crossings (Menasco balls) provides an endpoint for four segments of $P$. Since each segment has two endpoints, we must have $2 n$ segments and thus $4 n$ boundary segments. Since $P$ is alternating, each segment must bound part of an overdisk at one end and part of an underdisk at the other. The statement thus holds for $r=0$.

Now, suppose we add the realternating arcs of $A$ one at a time. Every time we add an arc, that arc has two endpoints, each on some segment of $P$. Each endpoint divides its segment into two new segments, so each arc in $A$ increases the number of segments by two. Thus, we end up with $2 n+2 r$ segments. Because each arc in $A$ is realternating, each segment will still bound part of an overdisk at one end and part of an underdisk at the other.

Proposition 4.7 Each of the $2 n+2 r$ segments (and at least this many boundary segments) of $P^{*}$ contains an odd, and therefore positive, number of intersection arc endpoints on its interior.

Proof $P$ everywhere bounds either an overdisk or an underdisk and switches exactly where it contains an intersection arc endpoint. Since each segment of $P^{*}$ bounds an overdisk at one end and an underdisk at the other, it must switch an odd number of times. Therefore, it must contain an odd number of intersection arc endpoints. 
Proposition 4.8 $P^{*}$ divides $F$ into $n+r+2$ regions, each with at least two boundary segments on its boundary.

Proof First suppose $A=\varnothing$. If $n=0$ we have two regions, and each time we add a crossing we add a region. Thus, for arbitrary $n$ we have $n+2$ regions. Since each arc in $A$ will separate a region in two, thus increasing the number of regions by one, we conclude that $P^{*}$ divides $F$ into $n+r+2$ regions.

Clearly the boundary of every region must contain at least one boundary segment. Suppose the boundary of some region contains only one boundary segment. Then either the two endpoints occur at the same crossing, which contradicts the fact that $P$ is reduced, or both endpoints of a realternating arc in $A$ are on the same edge of a projection $n$-gon of $P$, contradicting the assumption that $S$ is meridianally essential and cleanly embedded, by Proposition 4.4.

Given $P^{*}$ as above, suppose that $R$ is a region of $F \backslash P^{*}$. Also, suppose that $R \cap S=B$, where $B$ is a (possibly empty) collection of intersection arcs. Consider $B$ as a subset of $R$. This creates a new regional picture of $R \subset F$, which reveals how $S$ intersects $R$.

If $B$ is nonempty, there exist at least two outermost components of $R \backslash B$; that is, ones whose boundary contains exactly one arc from $B$. Since no segment contains both endpoints of any intersection arc from $A$, the boundary of each outermost region contains portions of at least two segments of $P^{*}$.

\section{Main results}

The proof of Theorem 5.4 will proceed by induction on the number of crossings. First, however, we use the results of the previous section to prove a critical lemma, which will be helpful in reducing the $n$-crossing case to an $(n-1)$-crossing case.

We will say that a surface $S$ intersects a Menasco ball $M_{i}$ in a crossing band if $S \cap M_{i}$ consists of a disk bounded by the overstrand and understrand on $\partial M_{i}$ together with a pair of opposite arcs on the equator of $M_{i}$, as in Figures 28 and 29. The two possible configurations of a crossing band at a particular crossing correspond to the two pairs of opposite arcs on the Menasco ball's equator. Note that a surface with a crossing band is not in Menasco form.

Lemma 5.1 A meridianally essential surface $S$ spanning an alternating link $L$ can be isotoped relative to a given nontrivial Menasco projection $P$ to obtain a crossing band. 


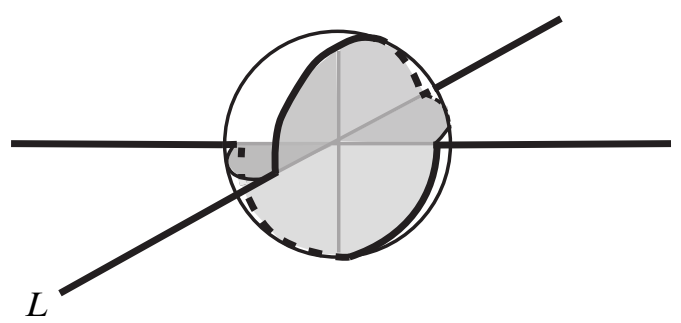

Figure 28: A crossing band in a Menasco ball
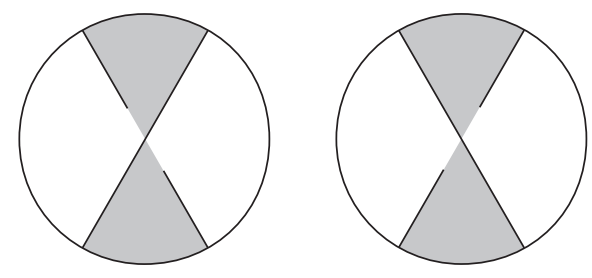

Figure 29: Crossing bands

Proof For contradiction, choose $S$ and $P$ so that no Menasco ball contains a crossing band and so that $S$ cannot be isotoped to make it so. Embed $S$ cleanly. No flat or dealternating intersection arc will be parallel to a crossing in $P$. Otherwise, we could have isotoped the neighborhood of that arc in $S$ into the parallel crossing, creating a crossing band, as in Figure 30.
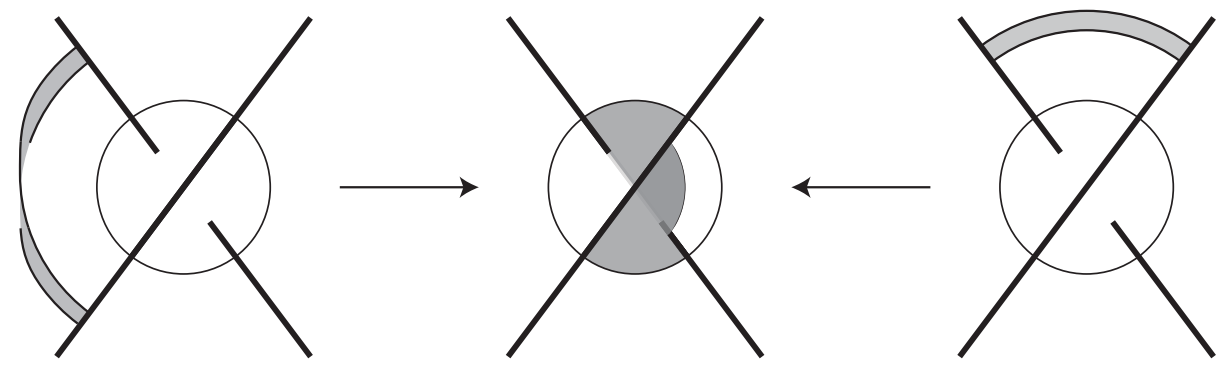

Figure 30: Isotoping neighborhoods of flat and dealternating arcs to obtain a crossing band

Therefore, the only type of intersection arc that can be parallel to a crossing in $P$ is a realternating arc. Let $A=\bigcup_{i=1}^{r} A_{i}$ be the collection of all realternating arcs in $S \cap F$ 
that are parallel to crossings. Note that two such arcs may be parallel to the same crossing, perhaps even on the same side of the crossing, thus making them parallel to each other.

Now consider $R$, one of the $n+r+2$ regions in $F \backslash P^{*}$, where $P^{*}=P \cup A$. Let $B=R \cap S$.

We claim that $\partial R$ contains at least two boundary segments which do not contain an endpoint of an arc in $B$. If $B$ is empty, this follows trivially, since the boundary of $R$ must contain at least two boundary segments, by Proposition 4.8. If $B$ is not empty, $R$ must contain at least two outermost subregions, each disjoint on its interior from $S$ and having a boundary consisting of exactly one arc in $B$ together with portions of at least two boundary segments of $P^{*}$.

Suppose the boundary of some outermost subregion $Q$ contains portions of exactly two boundary segments of $P^{*}$. Each of the endpoints of the arc in $B$ will lie on the interior of one of these boundary segments, so each boundary segment has exactly one endpoint on $\partial Q$. At these endpoints they will both connect either to the same arc in $A$ or to the same Menasco ball boundary, either of which will necessarily be parallel to the arc in $B$. Since all arcs in $A$ are parallel to crossings, the arc in $B$ must therefore be parallel to a crossing, giving a contradiction. Therefore, the boundary of every outermost subregion contains portions of at least three boundary segments of $P^{*}$. Two of these boundary segments will contain an endpoint of an $\operatorname{arc}$ in $B$ and will lie on the boundary of adjacent subregions as well. All other boundary segments will lie entirely on the boundary of this subregion and will not contain any intersection arc endpoints. Since either $B$ is empty or $R$ contains at least two outermost subregions, it follows that the boundary of every region of $F \backslash P^{*}$ contains at least two boundary arcs which contain no intersection arc endpoints.

Since $F \backslash P^{*}$ consists of $n+r+2$ regions, we have at least $2 n+2 r+4$ boundary segments which do not contain intersection arc endpoints. It follows that we have at most $(4 n+4 r)-(2 n+2 r+4)=2 n+2 r-4$ boundary segments (and certainly no more segments than this) that $d o$ contain intersection arc endpoints. Since each of the $2 n+2 r$ segments of $P^{*}$ must contain such an endpoint, we have a contradiction.

Corollary 5.2 Any spanning surface for a nonsplittable, alternating link is connected.

Proof This is immediate in the case of the trivial knot. Assume it to be true for all nonsplittable alternating links of no more than $n$ crossings. Let $L$ be a nonsplittable alternating link of $n+1$ crossings and let $S$ be a spanning surface. Then either $S$ is meridianally essential, or a series of compressions/meridianal boundary compressions 
takes $S$ to a meridianal essential spanning surface, $S^{\prime}$. Choose a reduced alternating projection of $L$, and put $S^{\prime}$ in Menasco form relative to that projection. Lemma 5.1 shows that $S^{\prime}$ can be isotoped to have a crossing that contains a crossing band. Cutting open the link and surface at that crossing results in a spanning surface $S^{\prime \prime}$ for a nonsplittable alternating link $L^{\prime}$ with fewer crossings. Note that nonsplittability of $L^{\prime}$ utilizes a theorem from Menasco [21]. Since $S^{\prime \prime}$ is connected, so is $S^{\prime}$ and hence $S$.

Lemma 5.3 Any surface $S$ spanning the unknot with a boundary slope of $2 k$ has $\chi=1-|k|-2 p$ for some $p \in \mathbb{N} \cup\{0\}$.

Proof Note that we have already said that the boundary slope of a spanning surface $S$ must be even. A spanning surface for the trivial knot can be thought of as a surface properly embedded in a solid torus $V$, which is the complement of the trivial knot. That surface will have a boundary on the torus boundary that wraps once meridianally and $2|k|$ times longitudinally around the torus. Compress the surface until it becomes an incompressible surface $S^{\prime}$. If $k=0$, the resulting surface must be a meridian disk. Otherwise, $S^{\prime}$ must be nonorientable, since the only incompressible orientable surface with one boundary component properly embedded in the solid torus is a disk.

By Tsau [35, Proposition 1, Corollary 3 and Remark 1], $S^{\prime}$ must have Euler characteristic $1-|k|$. Thus $S$ has Euler characteristic $\chi=1-|k|-2 p$ for some $p \in \mathbb{N} \cup\{0\}$.

Note also that a spanning surface for a knot is necessarily connected. We now proceed to the proof of the main theorem, which we restate here.

Theorem 5.4 Given an alternating projection $P(L)$ and a surface $S$ spanning $L$, we can construct a surface $T$ spanning $L$ with the same genus, orientability, and aggregate slope as $S$ such that $T$ is a basic state surface with respect to $P$, except perhaps at a collection of added crosscaps and/or handles. When $S$ is orientable, $T$ can be chosen to be orientable with respect to the orientation that $L$ inherits from $S$.

Proof We show this to be true for the trivial projection and proceed by induction on crossings in $P$.

Let $S$ be an orientable surface spanning the unknot. Since $S$ is orientable, either $\chi(S)=1$ or there exists a series of compressions $C_{1}, C_{2}, \ldots, C_{q}$ that takes $S$ to a disk. Since compression does not affect boundary slope, $S$ must have a slope of zero. The previous lemma then implies that $\chi(S)=1-2 p$. Adding $p$ handles to a disk then creates a spanning surface with the same Euler characteristic, slope, and orientability as $S$. 
Now suppose that $S$ is a nonorientable surface spanning the unknot with a slope of $2 k$ and an Euler characteristic of $1-|k|-2 p$. Then, adding to the disk $k+p$ of the appropriate type of crosscap and $p$ of the other type of crosscap constructs a surface with the same Euler characteristic, slope, and orientability as $S$. Thus, Theorem 5.4 holds for the trivial projection of the unknot.

We now proceed with the inductive proof of Theorem 5.4 assuming it to be true for all Menasco projections with fewer than $n$ crossings. If $P$ is not reduced, we can reduce it and apply our inductive hypothesis to get a surface with the same characteristics as $S$ that appears as a basic state surface, perhaps with a collection of added handles and/or crosscaps. We can then carry this surface along as we unreduce to $P$. Assume therefore that $P$ is reduced.

Suppose that our reduced projection $P$ has $n$ crossings and that $w(P)=y$ and $\operatorname{lk}(L)=z$. Also suppose that a surface $S$ spans $L$ with an aggregate slope of $l$ and an Euler characteristic of $x$. Recall that $\tau(S, P)=l-(y-2 z)$. Begin by performing a series of compressions/meridianal boundary compressions taking $S$ to a meridianally essential surface $S^{\prime}$, also spanning $L$. By Lemma 5.1, we can isotope $S^{\prime}$ to have a crossing band at a crossing in $P$. Without loss of generality, suppose that this is a positively twisted crossing. Cutting $S^{\prime}$ at this crossing as in Figure 31 produces a new surface $S^{*}$ spanning a link $L^{*}$ in an alternating projection $P^{*}$ with $n-1$ crossings such that $\tau\left(S^{*}, P^{*}\right)=l-(y-2 z)-1$ and $\chi\left(S^{*}\right)=x+1$. Notice that this projection may not be reduced. Reduce it to a projection $P^{* *}$.

By our inductive hypothesis, we can create a surface with the same characteristics as $S^{*}$ that appears state with respect to $P^{* *}$, except perhaps at a collection of added handles or crosscaps. We construct such a surface, stacking all inner disks above any outer disks containing them.

Call this surface $T^{*}$. We can then carry $T^{*}$ along as a state surface stacked in this manner while we unreduce from $P^{* *}$ to $P^{*}$. Recall that $\chi\left(T^{*}\right)=\chi\left(S^{*}\right)=x+1$. Also, since $T^{*}$ and $S^{*}$ have the same aggregate slope, $\tau\left(T^{*}, P^{*}\right)=\tau\left(S^{*}, P^{*}\right)=$ $l-(y-2 z)-1$. At the removed crossing, we then glue to $T^{*}$ a crossing band, which we know to be possible because of the particular layering of $T^{*}$. This gives a state surface $T^{\prime}$ spanning $L$ with $\chi\left(T^{\prime}\right)=(x+1)-1=x$ and $\tau(T, P)=[l-(y-2 z)-1]+1=$ $l-(y-2 z)$. Since $\tau\left(T^{\prime}, P\right)=\tau\left(S^{\prime}, P\right)$, we know that $l\left(T^{\prime}, L\right)=l\left(S^{\prime}, L\right)$. Adding the appropriate handles/crosscaps, corresponding to the initial compressions/meridianal boundary compressions of $S$ produces a surface $T$ spanning $L$ that appears state with respect to $P$, except at a collection of handles or crosscaps, and that has the same genus and aggregate slope as $S$. 


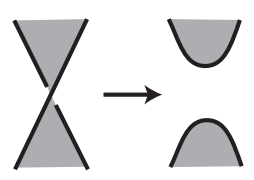

Figure 31: Cutting a crossing containing a crossing band yields a new surface.

The process can also be reversed.

It remains to show that $T$ has the same orientability as $S$. If $S^{*}$ and hence $T^{*}$ are nonorientable, then so are $S$ and $T$. Also, if there are any meridianal boundary compressions in going from $S$ to $S^{\prime}$, then both $S$ and $T$ are nonorientable. So assume instead that $S^{*}$ and $T^{*}$ are both orientable, and no meridianal boundary compressions occurred in going from $S$ to $S^{\prime}$. We have two cases to consider, as appearing in Figure 32, depending on the orientation of $L^{*}$ near the removed crossing band. In the case depicted in Figure 32(a), we can extend the orientation on $L^{*}$ to an orientation on $L$, and we can assign a normal direction to the crossing band as we glue it back in that agrees with the normal direction on both $S^{*}$ and $T^{*}$ to both sides of the crossing. Thus, both $S^{\prime}$ and $T^{\prime}$ will be orientable with respect to this orientation on $L$, as will $S$ and $T$. Similarly, as in the case depicted in Figure 32(b), if we were to glue in an untwisted band to both $S^{*}$ and to $T^{*}$ as appears in Figure 32(c), we would obtain a pair of orientable surfaces. Corollary 5.2 implies that each such surface contains an annulus that passes through this untwisted band once. Replacing the untwisted band with the actual crossing band to obtain $S^{\prime}$ and $T^{\prime}$ will result in the fact each contains a Mobius band, implying that both $S^{\prime}$ and $T^{\prime}$ are nonorientable, and therefore implying the same for $S$ and $T$.

(a)

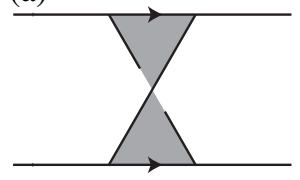

(b)

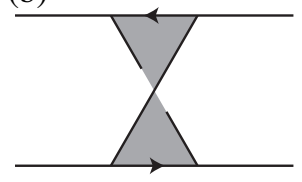

(c)

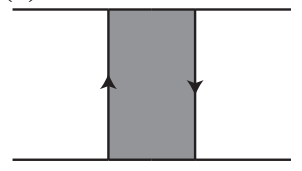

Figure 32: (a) and (b): Up to symmetry, the two possibilities for the orientation on $L$ near the removed crossing band. (c): In the case of (b), gluing an untwisted band extends the orientation on $L^{*}$ to one on $L$.

Thus, we have proven that our inductive construction preserves orientability as well as the Euler characteristic and aggregate slope.

All we have left to show is that our state surface $T$ can be a basic state surface (potentially with added crosscaps or handles), one in which no crossing connects a circle to itself. Suppose $T$ is a nonbasic state surface such that in its construction, we 
stacked all inner disks above outer ones, as described earlier. $T$ is then meridianally boundary-compressible. Performing this meridianal boundary compression will take $T$ to a state surface in which fewer crossings connect any circle to itself than in $T$. Performing in turn all possible meridianal boundary compressions of this sort and pairing each with the addition of an appropriate crosscap will create a new state surface (with added crosscaps and perhaps handles as well) that is in fact basic and that has the same Euler characteristic, aggregate slope, and orientability as $T$ and therefore as $S$.

\section{Implications}

We now provide some corollaries to Theorem 5.4.

Corollary 6.1 Given an alternating projection of a link, the minimal genus basic state surface (orientable or nonorientable) generated from that projection is a minimal genus spanning surface for that link. If a minimal genus basic state surface is nonorientable, it realizes the nonorientable genus for the link. If all minimal genus basic state surfaces are orientable, the nonorientable genus is $\frac{1}{2}$ greater than this minimal genus.

Corollary 6.2 There exists a surface $S$ spanning $L$ in an alternating projection $P$ with Euler characteristic $x$ and aggregate slope $l$ if and only if there exists a basic state surface $T$ generated from $P$ with Euler characteristic $x^{*}$ and aggregate slope $l^{*}$ such that $x=x^{*}+\left|l-l^{*}\right| / 2+2 p$ for some $p \in \mathbb{N} \cup\{0\}$. Furthermore, $S$ can be nonorientable if and only if either such a $T$ is nonorientable, $l \neq l^{\prime}$, or $p>0$. $S$ can be orientable if and only if such a $T$ is orientable and $l=l^{*}$.

Mizuma and Tsutsumi [26] point out that no example is known of two mutant knots with distinct crosscap number, unlike what occurs for orientable genus, where the KinoshitaTerasaka mutants provide just such an example. It is known that for alternating knots and links, orientable genus is preserved by mutation.

Corollary 6.3 If a knot is alternating, any mutant of it has the same crosscap number as it does.

Proof This follows from Theorem 5.4 together with the fact that a mutant of an alternating knot is also alternating, and must come from a sequence of mutations. Each of the mutations is along a 4-punctured sphere that intersects the projection plane in either a single circle intersecting the knot at four points or in two circles, each 


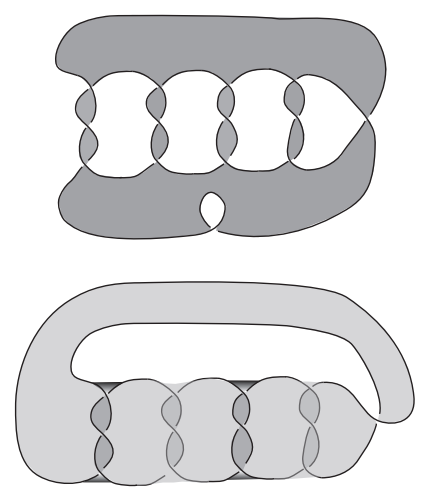

Figure 33: An example of a knot such that it has minimal genus nonorientable surfaces, one that is boundary-compressible and one that is boundaryincompressible

intersecting the knot at two points such that the sphere has two saddles. This follows from Menasco [21]. The collection of genera paired with orientability of spanning surfaces of the knot is preserved by mutancy along such 4-punctured spheres.

Hatcher and Thurston [15] proved that a 2-bridge knot cannot have two minimal nonorientable genus spanning surfaces, one boundary-incompressible and one boundarycompressible. They ask whether or not this is true in general. We answer that question in the negative with the following example.

Example Consider the knot and surfaces that appear in Figure 33. By applying the algorithm of Section 3, we find that a spanning surface $S$ with greatest possible Euler characteristic is orientable of genus 2 , and therefore the nonorientable genus is $\frac{5}{2}$, a surface for which can be obtained by adding a crosscap to the surface $S$. This surface $S_{1}$, which appears at the top of Figure 33, is incompressible, as any compression would yield a spanning surface with genus less than the minimum 2, and it is obviously boundary-compressible. However, we can also obtain the second surface $S_{2}$ appearing in Figure 33 by sliding one strand around the far side of the projection sphere and changing one crossing split. It is a nonbasic state surface. By situating the bands connecting top and bottom to be alternately over and under the main disk, we obtain a surface that is boundary-incompressible.

Lemma 6.4 The surface $S_{2}$ is incompressible and boundary-incompressible.

Proof That the surface is incompressible follows immediately from the fact that a compression would yield a spanning surface of genus less than 2 , contradicting the 


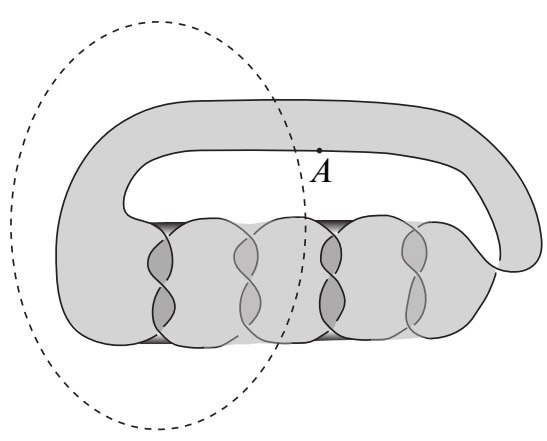

Figure 34: Proving the boundary-incompressibility of $S_{2}$

fact that 2 is the minimal genus possible for this knot. To see that it is boundaryincompressible, we intersect it with a sphere $Q$ intersecting the projection plane in the circle shown in Figure 34.

Suppose $S_{2}$ is boundary compressible. We can choose a boundary-compressing disk $D$ so that the two points of intersection of its boundary with $\partial S_{2}$ occur at or very near the point $A$ in the figure and so that it intersects $Q$ in a minimal number of components. Then, an outermost intersection arc of $D \cap Q$ on $D$ cuts a disk $D^{\prime}$ from $D$, the boundary of which is made up of an arc in $Q$ and an $\operatorname{arc}$ in $\partial N(K) \cup S_{2}$. This implies that to one of the sides of $Q$, the interior of this disk $D^{\prime}$ lies in the interior of a handlebody $H$ obtained by removing $S_{2} \cup N(K)$ from one of the balls bounded by $Q$. The boundary of $D^{\prime}$ consists of a single arc in $Q$ and a second arc, for which there are three cases. The second arc is either entirely contained in $S_{2}$, entirely contained in $\partial N(K)$, or it is made up of two arcs, one in $S_{2}$ and one in $\partial N(K)$. Because the fundamental group of a handlebody is a free product, in the first two cases, the arc in $\partial D^{\prime} \backslash\left(\partial D^{\prime} \cap Q\right)$ cannot pass over either of the two bands formed from the triple half-twists without immediately turning around and retracing its path, and it must therefore be isotopic on $S_{2} \cup \partial N(K)$ to a path that passes directly from left to right, beginning and ending on $Q$. However, the two twisted bands prevent such an arc from being isotopic into $Q$ while fixing its endpoints.

In the third case, the fact we can only jump once from $S_{2}$ to $\partial N(K)$ as we travel around $\partial D^{\prime}$ and those jumps occur in the vicinity of the point $A$, again implies that the triple twisting of the two bands prevents the arc $\partial D^{\prime} \backslash\left(\partial D^{\prime} \cap Q\right)$ from traversing one of the triple-twisted bands. Again, the arc must be isotopic on $S_{2} \cup \partial N(K)$ to a path that passes directly from left to right, beginning and ending on $Q$, the twisted bands of which again prevent it from being isotopic into $Q$ while fixing its endpoints, thereby proving $S_{2}$ is boundary-incompressible. 


\section{Appendix}

We conclude with a list of nonorientable genera of prime alternating knots through 10 crossings and 2-component links through 8 crossings. (Here $2{ }_{1}{ }^{2}:=2_{1}^{2}$ for space.)

\begin{tabular}{|c|c|c|c|c|c|c|c|c|c|c|c|}
\hline Knot & n. g. & Knot & n. g. & Knot & n. g. & Knot & n. g. & Knot & n.g. & Link & n. $\mathrm{g}$. \\
\hline $3_{1}$ & $1 / 2$ & $9_{9}$ & $3 / 2$ & $10_{8}$ & 1 & $10_{48}$ & $3 / 2$ & $10_{88}$ & $5 / 2$ & $21^{2}$ & $1 / 2$ \\
\hline $4_{1}$ & 1 & $9_{10}$ & $3 / 2$ & $10_{9}$ & $3 / 2$ & $10_{49}$ & 2 & $10_{89}$ & $5 / 2$ & $4_{1}^{2}$ & $1 / 2$ \\
\hline $5_{1}$ & $1 / 2$ & $9_{11}$ & $3 / 2$ & $10_{10}$ & $3 / 2$ & $10_{50}$ & $3 / 2$ & $10_{90}$ & 2 & $5_{1}^{2}$ & $1 / 2$ \\
\hline $5_{2}$ & 1 & $9_{12}$ & $3 / 2$ & $10_{11}$ & $3 / 2$ & $10_{51}$ & 2 & $10_{91}$ & 2 & $6_{1}{ }^{2}$ & $1 / 2$ \\
\hline $6_{1}$ & 1 & $9_{13}$ & $3 / 2$ & $10_{12}$ & $3 / 2$ & $10_{52}$ & $3 / 2$ & $10_{92}$ & 2 & $6_{2}{ }^{2}$ & $1 / 2$ \\
\hline 62 & 1 & $9_{14}$ & $3 / 2$ & $10_{13}$ & 2 & $10_{53}$ & 2 & $10_{93}$ & $3 / 2$ & $6_{3}{ }^{2}$ & 1 \\
\hline $6_{3}$ & $3 / 2$ & $9_{15}$ & 2 & $10_{14}$ & 2 & $10_{54}$ & $3 / 2$ & $10_{94}$ & 2 & $7_{1}^{2}$ & $1 / 2$ \\
\hline $7_{1}$ & $1 / 2$ & $9_{16}$ & $3 / 2$ & $10_{15}$ & $3 / 2$ & $10_{55}$ & 2 & $10_{95}$ & $5 / 2$ & $7_{2}^{2}$ & 1 \\
\hline 72 & 1 & $9_{17}$ & $3 / 2$ & $10_{16}$ & $3 / 2$ & $10_{56}$ & 2 & $10_{96}$ & 2 & $73^{2}$ & 1 \\
\hline 73 & 1 & $9_{18}$ & 2 & $10_{17}$ & $3 / 2$ & $10_{57}$ & $5 / 2$ & $10_{97}$ & 2 & $7_{4}{ }^{2}$ & $1 / 2$ \\
\hline 74 & $3 / 2$ & $9_{19}$ & 2 & $10_{18}$ & 2 & $10_{58}$ & 2 & $10_{98}$ & 2 & $75^{2}$ & 1 \\
\hline 75 & $3 / 2$ & 920 & $3 / 2$ & $10_{19}$ & $3 / 2$ & $10_{59}$ & 2 & $10_{99}$ & 2 & $76^{2}$ & 1 \\
\hline $7_{6}$ & $3 / 2$ & $9_{21}$ & 2 & $10_{20}$ & $3 / 2$ & $10_{60}$ & 2 & $10_{100}$ & $3 / 2$ & $8_{1}^{2}$ & $1 / 2$ \\
\hline 77 & $3 / 2$ & $9_{22}$ & $3 / 2$ & $10_{21}$ & $3 / 2$ & $10_{61}$ & 1 & $10_{101}$ & 2 & $8_{2}^{2}$ & $1 / 2$ \\
\hline $8_{1}$ & 1 & $9_{23}$ & 2 & $10_{22}$ & $3 / 2$ & $10_{62}$ & $3 / 2$ & $10_{102}$ & 2 & $83^{2}$ & 1 \\
\hline $8_{2}$ & 1 & $9_{44}$ & 2 & $10_{23}$ & 2 & $10_{63}$ & 2 & $10_{103}$ & 2 & $8_{4}^{2}$ & 1 \\
\hline $8_{3}$ & $3 / 2$ & $9_{25}$ & 2 & $10_{24}$ & 2 & $10_{64}$ & $3 / 2$ & $10_{104}$ & 2 & $85^{2}$ & 1 \\
\hline 84 & 1 & $9_{26}$ & 2 & $10_{25}$ & 2 & $10_{65}$ & 2 & $10_{105}$ & 2 & $86^{2}$ & 1 \\
\hline $8_{5}$ & 1 & $9_{27}$ & 2 & $10_{26}$ & 2 & $10_{66}$ & 2 & $10_{106}$ & 2 & $87^{2}$ & 1 \\
\hline $8_{6}$ & $3 / 2$ & $9_{28}$ & 2 & $10_{27}$ & 2 & $10_{67}$ & 2 & $10_{107}$ & $5 / 2$ & $88^{2}$ & $3 / 2$ \\
\hline 87 & $3 / 2$ & $9_{29}$ & $3 / 2$ & $10_{28}$ & $3 / 2$ & $10_{68}$ & $3 / 2$ & $10_{108}$ & $3 / 2$ & $89^{2}$ & 1 \\
\hline $8_{8}$ & $3 / 2$ & $9_{30}$ & 2 & $10_{29}$ & 2 & $10_{69}$ & $5 / 2$ & $10_{109}$ & $5 / 2$ & $8_{10^{2}}$ & 1 \\
\hline 89 & $3 / 2$ & $9_{31}$ & 2 & $10_{30}$ & 2 & $10_{70}$ & 2 & $10_{110}$ & 2 & $8_{11}^{2}$ & 1 \\
\hline 810 & $3 / 2$ & $9_{32}$ & 2 & $10_{31}$ & 2 & $10_{71}$ & $5 / 2$ & $10_{111}$ & 2 & $812^{2}$ & $3 / 2$ \\
\hline $8_{11}$ & $3 / 2$ & $9_{33}$ & 2 & $10_{32}$ & 2 & $10_{72}$ & 2 & $10_{112}$ & 2 & $8_{13^{2}}$ & $3 / 2$ \\
\hline $8_{12}$ & 2 & $9_{34}$ & 2 & $10_{33}$ & 2 & $10_{73}$ & $5 / 2$ & $10_{113}$ & $5 / 2$ & $8_{14}{ }^{2}$ & $3 / 2$ \\
\hline $8_{13}$ & $3 / 2$ & $9_{35}$ & $3 / 2$ & $10_{34}$ & $3 / 2$ & $10_{74}$ & 2 & $10_{114}$ & 2 & & \\
\hline 814 & 2 & $9_{36}$ & $3 / 2$ & $10_{35}$ & 2 & $10_{75}$ & 2 & $10_{115}$ & $5 / 2$ & & \\
\hline 815 & 2 & $9_{37}$ & 2 & $10_{36}$ & 2 & $10_{76}$ & 2 & $10_{116}$ & 2 & & \\
\hline $8_{16}$ & $3 / 2$ & $9_{38}$ & 2 & $10_{37}$ & 2 & $10_{77}$ & 2 & $10_{117}$ & $5 / 2$ & & \\
\hline $8_{17}$ & 2 & $9_{39}$ & 2 & $10_{38}$ & 2 & $10_{78}$ & 2 & $10_{118}$ & $5 / 2$ & & \\
\hline 818 & 2 & $9_{40}$ & 2 & $10_{39}$ & 2 & $10_{79}$ & 2 & $10_{119}$ & 2 & & \\
\hline $9_{1}$ & $1 / 2$ & $9_{41}$ & $3 / 2$ & $10_{40}$ & $5 / 2$ & $10_{80}$ & 2 & $10_{120}$ & $5 / 2$ & & \\
\hline $9_{2}$ & 1 & $10_{1}$ & 1 & $10_{41}$ & 2 & $10_{81}$ & $5 / 2$ & $10_{121}$ & $5 / 2$ & & \\
\hline $9_{3}$ & 1 & $10_{2}$ & 1 & $10_{42}$ & $5 / 2$ & $10_{82}$ & 2 & $10_{122}$ & 2 & & \\
\hline $9_{4}$ & 1 & $10_{3}$ & $3 / 2$ & $10_{43}$ & 2 & $10_{83}$ & 2 & $10_{123}$ & $5 / 2$ & & \\
\hline $9_{5}$ & $3 / 2$ & $10_{4}$ & 1 & $10_{44}$ & 2 & $10_{84}$ & $5 / 2$ & & & & \\
\hline 96 & $3 / 2$ & $10_{5}$ & $3 / 2$ & $10_{45}$ & $5 / 2$ & $10_{85}$ & $3 / 2$ & & & & \\
\hline 97 & $3 / 2$ & $10_{6}$ & $3 / 2$ & $10_{46}$ & 1 & $10_{86}$ & 2 & & & & \\
\hline $9_{8}$ & $3 / 2$ & $10_{7}$ & $3 / 2$ & $10_{47}$ & $3 / 2$ & $10_{87}$ & 2 & & & & \\
\hline
\end{tabular}

Table 1: Nonorientable genus for prime alternating knots through ten crossings and two-component links through eight crossings 


\section{References}

[1] C C Adams, The knot book: An elementary introduction to the mathematical theory of knots, W. H. Freeman, New York (1994) MR1266837

[2] C C Adams, Toroidally alternating knots and links, Topology 33 (1994) 353-369 MR1273788

[3] C Adams, Noncompact Fuchsian and quasi-Fuchsian surfaces in hyperbolic 3manifolds, Algebr. Geom. Topol. 7 (2007) 565-582 MR2308957

[4] C C Adams, J F Brock, J Bugbee, et al, Almost alternating links, Topology Appl. 46 (1992) 151-165 MR1184114

[5] C Adams, R Dorman, K Foley, J Kravis, S Payne, Alternating graphs, J. Combin. Theory Ser. B 77 (1999) 96-120 MR1710534

[6] C Adams, T Fleming, M Levin, A M Turner, Crossing number of alternating knots in $S \times I$, Pacific J. Math. 203 (2002) 1-22 MR1895923

[7] B A Burton, M Ozlen, Computing the crosscap number of a knot using integer programming and normal surfaces, ACM Trans. Math. Software 39 (2012) 18 MR3002773

[8] B E Clark, Crosscaps and knots, Internat. J. Math. Math. Sci. 1 (1978) 113-123 MR0478131

[9] R Crowell, Genus of alternating link types, Ann. of Math. 69 (1959) 258-275 MR0099665

[10] C L Curtis, S J Taylor, The Jones polynomial and boundary slopes of alternating knots, J. Knot Theory Ramifications 20 (2011) 1345-1354 MR2851712

[11] D Futer, Fiber detection for state surfaces arXiv:1201.1643

[12] D Futer, E Kalfagianni, J S Purcell, Quasifuchsian state surfaces arXiv 1209. 5719

[13] D Futer, E Kalfagianni, J Purcell, Guts of surfaces and the colored Jones polynomial, Lecture Notes in Mathematics 2069, Springer, Heidelberg (2013) MR3024600

[14] D Gabai, Genera of the alternating links, Duke Math. J. 53 (1986) 677-681 MR860665

[15] A Hatcher, W Thurston, Incompressible surfaces in 2-bridge knot complements, Invent. Math. 79 (1985) 225-246 MR778125

[16] C Hayashi, Links with alternating diagrams on closed surfaces of positive genus, Math. Proc. Cambridge Philos. Soc. 117 (1995) 113-128 MR1297898

[17] M Hirasawa, M Teragaito, Crosscap numbers of 2-bridge knots, Topology 45 (2006) 513-530 MR2218754

[18] J Hoste, M Thistlethwaite, J Weeks, The first 1,701,936 knots, Math. Intelligencer 20 (1998) 33-48 MR1646740 
[19] K Ichihara, S Mizushima, Crosscap numbers of pretzel knots, Topology Appl. 157 (2010) 193-201 MR2556097

[20] T W Mattman, O Sizemore, Bounds on the crosscap number of torus knots, J. Knot Theory Ramifications 16 (2007) 1043-1051 MR2364888

[21] W Menasco, Closed incompressible surfaces in alternating knot and link complements, Topology 23 (1984) 37-44 MR721450

[22] W Menasco, M Thistlethwaite, A geometric proof that alternating knots are nontrivial, Math. Proc. Cambridge Philos. Soc. 109 (1991) 425-431 MR1094743

[23] W W Menasco, M B Thistlethwaite, The Tait flyping conjecture, Bull. Amer. Math. Soc. 25 (1991) 403-412 MR1098346

[24] W W Menasco, M B Thistlethwaite, Surfaces with boundary in alternating knot exteriors, J. Reine Angew. Math. 426 (1992) 47-65 MR1155746

[25] W Menasco, M Thistlethwaite, The classification of alternating links, Ann. of Math. 138 (1993) 113-171 MR1230928

[26] Y Mizuma, Y Tsutsumi, Crosscap number, ribbon number and essential tangle decompositions of knots, Osaka J. Math. 45 (2008) 391-401 MR2441946

[27] H Murakami, A Yasuhara, Crosscap number of a knot, Pacific J. Math. 171 (1995) 261-273 MR1362987

[28] K Murasugi, On the genus of the alternating knot, I, J. Math. Soc. Japan 10 (1958) 94-105 MR0099664

[29] K Murasugi, On the genus of the alternating knot, II, J. Math. Soc. Japan 10 (1958) 235-248 MR0099664

[30] M Ozawa, Essential state surfaces for knots and links, J. Aust. Math. Soc. 91 (2011) 391-404 MR2900614

[31] M D Pabiniak, J H Przytycki, R Sazdanović, On the first group of the chromatic cohomology of graphs, Geom. Dedicata 140 (2009) 19-48 MR2504733

[32] S Rankin, O Flint, J Schermann, Enumerating the prime alternating knots, I, J. Knot Theory Ramifications 13 (2004) 57-100 MR2039118

[33] S Rankin, O Flint, J Schermann, Enumerating the prime alternating knots, II, J. Knot Theory Ramifications 13 (2004) 101-149 MR2039119

[34] M Teragaito, Crosscap numbers of torus knots, Topology Appl. 138 (2004) 219-238 MR2035482

[35] CM Tsau, A note on incompressible surfaces in solid tori and in lens spaces, from: “Knots 90”, (A Kawauchi, editor), de Gruyter, Berlin (1992) 213-229 MR1177425

[36] T Tsukamoto, A criterion for almost alternating links to be non-splittable, Math. Proc. Cambridge Philos. Soc. 137 (2004) 109-133 MR2075045 
[37] G Zhang, Crosscap numbers of two-component links, Kyungpook Math. J. 48 (2008) 241-254 MR2429312

Mathematics and Statistics Department, Bronfman Science Center

Williams College, Williamstown, MA 01267, USA

Department of Mathematics, University of Iowa

14 MacLean Hall, Iowa City, IA 52242-1419, USA

Colin.C.Adams@williams.edu, thomas.kindred@gmail.com

http://www.williams . edu/go/math/cadams/

Received: 21 February 2012 Revised: 29 January 2013 